\title{
ON NEW CLASSES OF NONNEGATIVE SYMMETRIC TENSORS*
}

\author{
BILIAN CHEN ${ }^{\dagger}$, SIMAI HE ${ }^{\ddagger}$, ZHENING LI§ ${ }^{\S}$ AND SHUZHONG ZHANG
}

\begin{abstract}
In this paper we introduce three new classes of nonnegative forms (or equivalently, symmetric tensors) and their extensions. The newly identified nonnegative symmetric tensors constitute distinctive convex cones in the space of general symmetric tensors (order six or above). For the special case of quartic forms, they collapse into the set of convex quartic homogeneous polynomial functions. We discuss the properties and applications of the new classes of nonnegative symmetric tensors in the context of polynomial and tensor optimization. Numerical experiments for solving certain polynomial optimization models based on the new classes of nonnegative symmetric tensors are presented.
\end{abstract}

Key words. symmetric tensors, nonnegative forms, polynomial and tensor optimization

AMS subject classifications. 15A69, 12Y05, 90C26

DOI. $10.1137 / 140988796$

1. Introduction. A classical result originally due to Banach [3] states that if $L\left(\boldsymbol{x}^{1}, \boldsymbol{x}^{2}, \ldots, \boldsymbol{x}^{m}\right)$ is a continuous symmetric $m$-linear form, then

$$
\begin{aligned}
& \sup \left\{\left|L\left(\boldsymbol{x}^{1}, \boldsymbol{x}^{2}, \ldots, \boldsymbol{x}^{m}\right)\right| \mid\left\|\boldsymbol{x}^{1}\right\| \leq 1,\left\|\boldsymbol{x}^{2}\right\| \leq 1, \ldots,\left\|\boldsymbol{x}^{m}\right\| \leq 1\right\} \\
& \quad=\sup \{|L(\boldsymbol{x}, \boldsymbol{x}, \ldots, \boldsymbol{x})|\|\| \boldsymbol{x} \| \leq 1\} .
\end{aligned}
$$

One is referred to [32] for a recent proof. An extension of the above result can be found in [8]. Although the result holds for the Banach space (where $\boldsymbol{x}$ resides) in general, for our purpose in this paper it is useful to consider $\boldsymbol{x}$ to be in an $n$-dimensional Euclidean space. In the latter case, $L(\boldsymbol{x}, \boldsymbol{x}, \ldots, \boldsymbol{x})$ becomes a homogenous polynomial of $n$ variables and degree $m$, whose coefficients form nothing but a symmetric tensor in $\mathbb{R}^{n^{m}}$. By symmetry (also known as supersymmetry in some papers in the literature), we mean that the entries of the tensor are invariant under the permutation of its indices. In the tensor setting, (1.1) is essentially equivalent to the fact that the best rank-one approximation of a symmetric tensor can be obtained at a symmetric rankone tensor, which was recently rediscovered in $[8,40]$.

Two natural questions arise in (1.1): (i) Can the absolute-value sign be removed? (ii) Can the constraint $\|\boldsymbol{x}\| \leq 1$ be replaced by some more general constraints? The

* Received by the editors September 25, 2014; accepted for publication (in revised form) November 1, 2016; published electronically February 23, 2017.

http://www.siam.org/journals/siopt/27-1/98879.html

Funding: Research of the first author was supported in part by National Natural Science Foundation of China (grant 11301436). Research of the second author was supported in part by National Natural Science Foundation of China (grant 71440014), Program for Professor of Special Appointment (Eastern Scholar) at Shanghai Institutions of Higher Learning, and Program for Innovative Research Team of Shanghai University of Finance and Economics. Research of the fourth author was supported in part by National Science Foundation of USA (grant CMMI-1462408).

${ }^{\dagger}$ Department of Automation, Xiamen University, Xiamen 361005, China (blchen@xmu.edu.cn).

${ }^{\ddagger}$ School of Information Management and Engineering, Shanghai University of Finance and Economics, Shanghai 200433, China (simaihe@mail.shufe.edu.cn).

$\S$ Department of Mathematics, University of Portsmouth, Portsmouth PO1 3HF, United Kingdom (zheningli@gmail.com).

IDepartment of Industrial and Systems Engineering, University of Minnesota, Minneapolis, MN 55455 (zhangs@umn.edu). 
answer to these questions would be negative without further conditions. This, in fact, triggers an interesting question: What kind of $m$-form $L$ will naturally ensure that

$$
\begin{aligned}
& \sup \left\{L\left(\boldsymbol{x}^{1}, \boldsymbol{x}^{2}, \ldots, \boldsymbol{x}^{m}\right) \mid \boldsymbol{x}^{1} \in S, \boldsymbol{x}^{2} \in S, \ldots, \boldsymbol{x}^{m} \in S\right\} \\
& \quad=\sup \{L(\boldsymbol{x}, \boldsymbol{x}, \ldots, \boldsymbol{x}) \mid \boldsymbol{x} \in S\} ?
\end{aligned}
$$

By taking $m=2$ as an example, one quickly finds out that if $L\left(\boldsymbol{x}^{1}, \boldsymbol{x}^{2}\right)=\left(\boldsymbol{x}^{1}\right)^{\mathrm{T}} Q \boldsymbol{x}^{2}$, then the above holds for all $S \subseteq \mathbb{R}^{n}$ if and only if $Q$ is positive semidefinite. In case $m=4$, as we will later discuss in the paper, the necessary and sufficient condition for the equivalence is that the quartic form $L(\boldsymbol{x}, \boldsymbol{x}, \boldsymbol{x}, \boldsymbol{x})$ is convex. Surprisingly, further investigation into the case $m \geq 6$ reveals a new structure of the forms or symmetric tensors, which will be termed $M$-quasiconvex in this paper.

The new notion of M-quasiconvexity is not only theoretically interesting, but also useful for solving practical polynomial and tensor optimization models. For instance, polynomial optimization with spherical constraint is important and widely studied in the literature (see, e.g., $[11,8]$ ), and is closely related to the computation of eigenvalues and singular values of tensors proposed by Lim [24] and Qi [35] independently. In [8], we proposed a new convergent solution method for block optimization models, known as the maximum block improvement (MBI). It turns out that the MBI method works well for polynomial optimization with spherical constraint. Essentially, in [8] homogeneous polynomial optimization over spherical constraint is relaxed to the corresponding multilinear form optimization. Two questions arise in this context: (i) When will the relaxation be exact? (ii) After one finds a stationary solution to the relaxed problem, how can a solution to the original problem be obtained? The results in the current paper help to address these issues.

Our analysis relies on the notion of nonnegative polynomials or nonnegative tensors. In fact, there is a known intrinsic connection between the optimization of a polynomial function and the description of all polynomial functions which are nonnegative over a given domain. This connection was explored earlier by Sturm and Zhang [39] for the case of quadratic polynomials, and Luo, Sturm, and Zhang [25] for the case of biquadratic functions. We also refer to $[14,37,12,16,1]$ and a recent book [5] for investigating the relationship between nonnegative polynomials and sumof-sqaures polynomials. In general, for the nonnegativity of a polynomial function over a semialgebraic set defined by polynomial functions, there are classical representations based on sum of squares such as Putinar's Positivstellensatz [34] and Reznick [38]. These obviously connect to the sum-of-squares approach for general polynomial optimization problems; see e.g., [31, 21]. The final interesting connection that will be established in this study is the link to the convexity of a polynomial function. In fact, convexity of an even degree form is a stronger notion than mere nonnegativity. For instance, it is well known that a quartic form $L(\boldsymbol{x}, \boldsymbol{x}, \boldsymbol{x}, \boldsymbol{x})$ is convex if and only if it biquadratically nonnegative, i.e., $L(\boldsymbol{x}, \boldsymbol{x}, \boldsymbol{y}, \boldsymbol{y}) \geq 0$, hence nonnegative. In the world of higher (than 2) degree forms, checking the nonnegativity and convexity are both difficult tasks. For instance, Ahmadi et al. [1] proved that checking the convexity of a quartic form is actually strongly NP-hard in general, highlighting a crucial difference between quadratic forms and quartic forms. Speaking of quartic polynomials, in a recent study, Jiang, Li, and Zhang [16] discussed six fundamentally important convex cones of quartic forms, including the cone of nonnegative quartic forms, the sum of squared quartic forms, the convex quartic forms, and the sum of fourth powered linear forms. The complexity status of these nonnegative tensor cones are discussed as well. 
In this paper, we extend the study beyond quartic forms. We show that the world of quartic forms is still special, in that an interesting structure to be termed M-quasiconvexity in this paper is still hidden under the usual convexity. Applying the new notion of M-quasiconvexity and its properties, we establish an equivalence between any constrained homogeneous polynomial optimization and its multilinear tensor relaxation model. This enables us to apply some block coordinate search methods to solve the multilinear model, and also suggests a simple way to find a stationary solution for the original model. A new general scheme to solve certain classes of polynomial optimization models, named lift, relax, block-optimize, and compare (LRBC), is proposed. The paper is organized as follows. In section 2, we introduce the notations for tensors and polynomials, and define new classes of nonnegative tensors with illustrating examples. Sections 3, 4, and 5 are devoted to the theoretical study of these classes of tensors, i.e., their equivalent definitions, relationships and proper containments. As applications of the classes of tensors in polynomial and tensor optimization, we propose the LRBC scheme and discuss some practical examples in section 6 . Numerical experiments are presented in section 7 to further illustrate the importance of the classes of nonnegative tensors. Finally we conclude the paper in section 8 by discussing a generalization of the newly introduced nonnegative tensors.

2. Preparations. Throughout this paper we uniformly use nonbold lowercase letters, boldface lowercase letters, capital letters, and calligraphic letters to denote scalars, vectors, matrices, and tensors, respectively; e.g., a scalar $i$, a vector $\boldsymbol{x}$, a matrix $A$, and a tensor $\mathcal{F}$. We use subscripts to denote their components; e.g., $x_{i}$ is the $i$ th entry of a vector $\boldsymbol{x}, A_{i j}$ is the $(i, j)$ th entry of a matrix $A$, and $\mathcal{F}_{i j k}$ is the $(i, j, k)$ th entry of a third order tensor $\mathcal{F}$. As previously mentioned, an $m$ th order tensor $\mathcal{F} \in \mathbb{R}^{n_{1} \times n_{2} \times \cdots \times n_{m}}$ is symmetric if $n_{1}=n_{2}=\cdots=n_{m}(=n)$ and every component $\mathcal{F}_{i_{1} i_{2} \ldots i_{m}}$ is invariant under all permutations of $\left\{i_{1}, i_{2}, \ldots, i_{m}\right\}$; the set of such symmetric tensors is denoted by $\mathbb{S}^{n^{m}}$.

The symbol $\circ$ denotes the vector outer product. For example, for vectors $\boldsymbol{x} \in$ $\mathbb{R}^{n_{1}}, \boldsymbol{y} \in \mathbb{R}^{n_{2}}, \boldsymbol{z} \in \mathbb{R}^{n_{3}}$, the notion $\boldsymbol{x} \circ \boldsymbol{y} \circ \boldsymbol{z}$ represents a third order tensor $\mathcal{F} \in$ $\mathbb{R}^{n_{1} \times n_{2} \times n_{3}}$, where $\mathcal{F}_{i j k}=x_{i} y_{j} z_{k}$ for all $(i, j, k)$. The symbol $\otimes$ represents the matrix outer product. If tensor $\mathcal{F}=X \otimes X$ for some $X \in \mathbb{R}^{n \times n}$, then $\mathcal{F}_{i j k \ell}=X_{i j} X_{k \ell}$ for all $(i, j, k, \ell)$.

Given any $m$ th order tensor $\mathcal{F} \in \mathbb{R}^{n_{1} \times n_{2} \times \cdots \times n_{m}}$, we define $F$ to be its associated multilinear form, i.e.,

$$
\begin{aligned}
& F\left(\boldsymbol{x}^{1}, \boldsymbol{x}^{2}, \ldots, \boldsymbol{x}^{m}\right):=\sum_{1 \leq i_{1} \leq n_{1}, 1 \leq i_{2} \leq n_{2}, \ldots, 1 \leq i_{m} \leq n_{m}} \mathcal{F}_{i_{1} i_{2} \ldots i_{m}} x_{i_{1}}^{1} x_{i_{2}}^{2} \ldots x_{i_{m}}^{m}, \\
& =\left\langle\mathcal{F}, \boldsymbol{x}^{1} \circ \boldsymbol{x}^{2} \circ \cdots \circ \boldsymbol{x}^{m}\right\rangle \text {, }
\end{aligned}
$$

where $\boldsymbol{x}^{k} \in \mathbb{R}^{n_{k}}$ for $k=1,2, \ldots, m$ and $\langle\cdot, \cdot\rangle$ denotes the Frobenius inner product. Closely related to the multilinear form is a general $m$ th degree homogeneous polynomial $f(\boldsymbol{x})$ in variable $\boldsymbol{x} \in \mathbb{R}^{n}$, with its associated symmetric tensor $\mathcal{F} \in \mathbb{S}^{n^{m}}$. In fact, symmetric tensors are bijectively related to homogeneous polynomials; see [20]. Denoting $F$ to be the multilinear form associated with the symmetric tensor $\mathcal{F}$, we then have

$$
f(\boldsymbol{x})=F(\underbrace{\boldsymbol{x}, \boldsymbol{x}, \ldots, \boldsymbol{x}}_{m})=\sum_{1 \leq i_{1}, i_{2}, \ldots, i_{m} \leq n} \mathcal{F}_{i_{1} i_{2} \ldots i_{m}} x_{i_{1}} x_{i_{2}} \ldots x_{i_{m}}=\langle\mathcal{F}, \underbrace{\boldsymbol{x} \circ \boldsymbol{x} \circ \cdots \circ \boldsymbol{x}}_{m}\rangle .
$$


In this paper we uniformly use the 2-norm for vectors, matrices, and tensors in general, which is the usual Euclidean norm or the Frobenius norm. For example, the norm of an $m$ th order tensor $\mathcal{F} \in \mathbb{R}^{n_{1} \times n_{2} \times \cdots \times n_{m}}$ is defined as

$$
\|\mathcal{F}\|:=\sqrt{\langle\mathcal{F}, \mathcal{F}\rangle}=\sqrt{\sum_{1 \leq i_{1} \leq n_{1}, 1 \leq i_{2} \leq n_{2}, \ldots, 1 \leq i_{m} \leq n_{m}} \mathcal{F}_{i_{1} i_{2} \ldots i_{m}}{ }^{2}} .
$$

2.1. Nonnegativity and co-quadratic nonnegativity. All the discussion in this paper is focused on nonnegative tensors, which are the following.

DeFINITION 2.1. Suppose $F$ is a multilinear form associated with a $2 m$ th order symmetric tensor $\mathcal{F} \in \mathbb{S}^{n^{2 m}}$. The tensor $\mathcal{F}$ (or the form $F$ ) is called nonnegative if

$$
F(\underbrace{\boldsymbol{x}, \boldsymbol{x}, \ldots, \boldsymbol{x}}_{2 m}) \geq 0 \quad \forall \boldsymbol{x} \in \mathbb{R}^{n} .
$$

We emphasize that a symmetric tensor is exactly a symmetric multilinear form; the former is denoted by a calligraphic letter and the latter is denoted by its corresponding capital letter, for easy presentation, whenever appropriate. Therefore, any terminology related to a form is also related to its tensor representation, e.g., a nonnegative form is simply a nonnegative tensor.

As a notation, we denote the set of nonnegative symmetric tensors

$$
\mathbb{S}_{+}^{n^{2 m}}:=\left\{\mathcal{F} \in \mathbb{S}^{n^{2 m}} \mid F(\underbrace{\boldsymbol{x}, \boldsymbol{x}, \ldots, \boldsymbol{x}}_{2 m}) \geq 0 \forall \boldsymbol{x} \in \mathbb{R}^{n}\right\} .
$$

Obviously, the degree of a nonnegative form has to be even. It is well known that checking the nonnegativity of a quadratic form can be done in polynomial time, which amounts to checking the positive semidefiniteness of its associated symmetric matrix. However, it is NP-hard to check the nonnegativity of a form whose degree is larger than 2; see, e.g., [16]. To extend the definition of nonnegativity, let us introduce the following.

Definition 2.2. Suppose $F$ is a multilinear form associated with a $2 m$ th order symmetric tensor $\mathcal{F} \in \mathbb{S}^{n^{2 m}}$. The tensor $\mathcal{F}$ is called co-quadratic nonnegative if

$$
F\left(\boldsymbol{x}_{1}, \boldsymbol{x}_{1}, \boldsymbol{x}_{2}, \boldsymbol{x}_{2}, \ldots, \boldsymbol{x}_{m}, \boldsymbol{x}_{m}\right) \geq 0 \quad \forall \boldsymbol{x}_{1}, \boldsymbol{x}_{2}, \ldots, \boldsymbol{x}_{m} \in \mathbb{R}^{n} .
$$

Let us denote the set of co-quadratic nonnegative tensors to be

$$
\mathbb{S}_{2+}^{n^{2 m}}:=\left\{\mathcal{F} \in \mathbb{S}^{n^{2 m}} \mid F\left(\boldsymbol{x}_{1}, \boldsymbol{x}_{1}, \boldsymbol{x}_{2}, \boldsymbol{x}_{2}, \ldots, \boldsymbol{x}_{m}, \boldsymbol{x}_{m}\right) \geq 0 \forall \boldsymbol{x}_{1}, \boldsymbol{x}_{2}, \ldots, \boldsymbol{x}_{m} \in \mathbb{R}^{n}\right\} .
$$

Clearly, a co-quadratic nonnegative tensor is always nonnegative, i.e., $\mathbb{S}_{2+}^{n^{2 m}} \subseteq \mathbb{S}_{+}^{n^{2 m}}$. In particular, when $m=1$, the co-quadratic nonnegativity is equivalent to the nonnegativity of a quadratic form and, when $m=2$, the co-quadratic nonnegativity is equivalent to the convexity of a quartic form (see below).

Proposition 2.3. A co-quadratic nonnegative form is always convex. In particular, a quartic form is co-quadratic nonnegative if and only if it is convex.

Proof. It is straightforward to compute the Hessian matrix of a form $F(\underbrace{\boldsymbol{x}, \boldsymbol{x}, \ldots, \boldsymbol{x}}_{2 m})$, which is $2 m(2 m-1) F(\underbrace{\boldsymbol{x}, \boldsymbol{x}, \ldots, \boldsymbol{x}}_{2 m-2}, \cdot, \cdot)$. Therefore, the form is convex if and only if 
$F(\underbrace{\boldsymbol{x}, \boldsymbol{x}, \ldots, \boldsymbol{x}}_{2 m-2}, \cdot, \cdot)$ is a positive semidefinite matrix for all $\boldsymbol{x} \in \mathbb{R}^{n}$, which is equivalent to

$$
F(\underbrace{\boldsymbol{x}, \boldsymbol{x}, \ldots, \boldsymbol{x}}_{2 m-2}, \boldsymbol{y}, \boldsymbol{y}) \geq 0 \quad \forall \boldsymbol{x}, \boldsymbol{y} \in \mathbb{R}^{n} .
$$

By Definition 2.2, it is obvious that co-quadratic nonnegativity implies (2.1), which is convexity. In particular, for the case of quartic form $(m=2)$, co-quadratic nonnegativity is equivalent to convexity.

Therefore, when $m=2$, the usual nonnegative quartic form $F(\boldsymbol{x}, \boldsymbol{x}, \boldsymbol{x}, \boldsymbol{x})$ is not necessarily convex, while convexity implies nonnegativity. As a result, co-quadratic nonnegativity is indeed stronger than nonnegativity. When $m \geq 3$, co-quadratic nonnegativity is even stronger than convexity. Unfortunately, checking the co-quadratic nonnegativity for $m=2$ is also NP-hard [1]. Jiang, Li, and Zhang [16] presented a study on different classes of nonnegative quartic forms, for the case when $m=2$. Below we present two examples of co-quadratic nonnegative tensors for general $\mathrm{m}$.

Example 2.4. If $\boldsymbol{a}_{1}, \boldsymbol{a}_{2}, \ldots, \boldsymbol{a}_{s} \in \mathbb{R}^{n}$, then the tensor

$$
\mathcal{F}=\sum_{i=1}^{s} \underbrace{\boldsymbol{a}_{i} \circ \boldsymbol{a}_{i} \circ \cdots \circ \boldsymbol{a}_{i}}_{2 m}
$$

is co-quadratic nonnegative.

The reason for this is that for all $\boldsymbol{x}_{1}, \boldsymbol{x}_{2}, \ldots, \boldsymbol{x}_{m} \in \mathbb{R}^{n}$,

$$
F\left(\boldsymbol{x}_{1}, \boldsymbol{x}_{1}, \boldsymbol{x}_{2}, \boldsymbol{x}_{2}, \ldots, \boldsymbol{x}_{m}, \boldsymbol{x}_{m}\right)=\sum_{i=1}^{s}\left(\boldsymbol{a}_{i}^{\mathrm{T}} \boldsymbol{x}_{1}\right)^{2}\left(\boldsymbol{a}_{i}^{\mathrm{T}} \boldsymbol{x}_{2}\right)^{2} \ldots\left(\boldsymbol{a}_{i}^{\mathrm{T}} \boldsymbol{x}_{m}\right)^{2} \geq 0 .
$$

Example 2.5. The symmetric tensor associated with homogeneous polynomial $\left(\boldsymbol{x}^{\mathrm{T}} \boldsymbol{x}\right)^{m}$ is co-quadratic nonnegative. Explicitly, its multilinear form is

$$
F\left(\boldsymbol{x}_{1}, \boldsymbol{x}_{2}, \ldots, \boldsymbol{x}_{2 m}\right)=\frac{1}{|\Pi|} \sum_{\left(i_{1} i_{2} \ldots i_{2 m}\right) \in \Pi}\left(\boldsymbol{x}_{i_{1}}^{\mathrm{T}} \boldsymbol{x}_{i_{2}}\right)\left(\boldsymbol{x}_{i_{3}}^{\mathrm{T}} \boldsymbol{x}_{i_{4}}\right) \ldots\left(\boldsymbol{x}_{i_{2 m-1}}^{\mathrm{T}} \boldsymbol{x}_{i_{2 m}}\right)
$$

where $\Pi$ is the set of all permutations of $\{1,2, \ldots, 2 m\}$.

In fact for $m=1$, this symmetric tensor is nothing but an identity matrix, which is clearly co-quadratic nonnegative. For $m=2$, it is easy to verify that

$$
F\left(\boldsymbol{x}_{1}, \boldsymbol{x}_{1}, \boldsymbol{x}_{2}, \boldsymbol{x}_{2}\right)=\frac{1}{3}\left(\boldsymbol{x}_{1}^{\mathrm{T}} \boldsymbol{x}_{1}\right)\left(\boldsymbol{x}_{2}^{\mathrm{T}} \boldsymbol{x}_{2}\right)+\frac{2}{3}\left(\boldsymbol{x}_{1}^{\mathrm{T}} \boldsymbol{x}_{2}\right)^{2} \geq 0 \quad \forall \boldsymbol{x}_{1}, \boldsymbol{x}_{2} \in \mathbb{R}^{n} .
$$

For general $m \geq 3$, it is not an easy job to directly check the nonnegativity of $F\left(\boldsymbol{x}_{1}, \boldsymbol{x}_{1}, \boldsymbol{x}_{2}, \boldsymbol{x}_{2}, \ldots, \boldsymbol{x}_{m}, \boldsymbol{x}_{m}\right)$ using (2.2). However, this property can be verified thanks to the so-called Hilbert's identity (see, e.g., $[4,15]$ ), which states that there exist vectors $\boldsymbol{b}_{1}, \boldsymbol{b}_{2}, \ldots, \boldsymbol{b}_{s} \in \mathbb{R}^{n}$ such that

$$
\left(\boldsymbol{x}^{\mathrm{T}} \boldsymbol{x}\right)^{m}=\sum_{i=1}^{s}\left(\boldsymbol{b}_{i}^{\mathrm{T}} \boldsymbol{x}\right)^{2 m} \quad \forall \boldsymbol{x} \in \mathbb{R}^{n}
$$


Thus, the symmetric tensor $\mathcal{F}$ can be expressed by

$$
\mathcal{F}=\sum_{i=1}^{s} \underbrace{\boldsymbol{b}_{i} \circ \boldsymbol{b}_{i} \circ \cdots \circ \boldsymbol{b}_{i}}_{2 m},
$$

which is co-quadratic nonnegative as Example 2.4 stipulates.

2.2. M-quasiconvexity and co-quadratic M-quasiconvexity. For a given set of entry vectors, a tensor form naturally lends to some degree of freedom in distributing the multiplicities among these entry vectors. To capture the characteristic of how the distribution of the multiplicities affects the function values, let us introduce the following notion of M-quasiconvexity. Here the terminology is to be understood as "quasiconvexity in the multiplicity of its entries"; one should distinguish this notion from the so-called M-convex functions in the theory of discrete convex functions.

Definition 2.6. Suppose $F$ is a nonnegative form associated with a $2 m$ th order symmetric tensor $\mathcal{F} \in \mathbb{S}^{n^{2 m}}$. The tensor $\mathcal{F}$ is called $M$-quasiconvex if

$F(\underbrace{\boldsymbol{x}_{1}, \ldots, \boldsymbol{x}_{1}}_{\lambda_{1}}, \underbrace{\boldsymbol{x}_{2}, \ldots, \boldsymbol{x}_{2}}_{\lambda_{2}}, \ldots, \underbrace{\boldsymbol{x}_{s}, \ldots, \boldsymbol{x}_{s}}_{\lambda_{s}}) \leq \max _{1 \leq i \leq s}\left\{F(\underbrace{\boldsymbol{x}_{i}, \boldsymbol{x}_{i}, \ldots, \boldsymbol{x}_{i}}_{2 m})\right\} \quad \forall \boldsymbol{x}_{1}, \boldsymbol{x}_{2}, \ldots, \boldsymbol{x}_{s} \in \mathbb{R}^{n}$

for any positive integers $s$ and $\lambda_{i}(i=1,2, \ldots, s)$ with $\sum_{i=1}^{s} \lambda_{i}=2 m$.

It is easy to observe that an $\mathrm{M}$-quasiconvex $\mathcal{F}$ is actually equivalent to

$$
F\left(\boldsymbol{x}_{1}, \boldsymbol{x}_{2}, \ldots, \boldsymbol{x}_{2 m}\right) \leq \max _{1 \leq i \leq 2 m}\left\{F(\underbrace{\boldsymbol{x}_{i}, \boldsymbol{x}_{i}, \ldots, \boldsymbol{x}_{i}}_{2 m})\right\} \quad \forall \boldsymbol{x}_{1}, \boldsymbol{x}_{2}, \ldots, \boldsymbol{x}_{2 m} \in \mathbb{R}^{n},
$$

i.e., (2.3) holds for a special case when $s=2 m$ and $\lambda_{i}=1$ for $i=1,2, \ldots, 2 m$. Thus to verify the M-quasiconvexity, it is convenient to check only (2.4) rather than (2.3) for all combinations of $\lambda_{i}$ 's. For ease of referencing, let us denote

$$
\begin{aligned}
\mathbb{M}^{n^{2 m}}:=\left\{\mathcal{F} \in \mathbb{S}_{+}^{n^{2 m}} \mid\right. & F\left(\boldsymbol{x}_{1}, \boldsymbol{x}_{2}, \ldots, \boldsymbol{x}_{2 m}\right) \\
& \left.\leq \max _{1 \leq i \leq 2 m}\left\{F(\underbrace{\boldsymbol{x}_{i}, \boldsymbol{x}_{i}, \ldots, \boldsymbol{x}_{i}}_{2 m})\right\} \forall \boldsymbol{x}_{1}, \boldsymbol{x}_{2}, \ldots, \boldsymbol{x}_{2 m} \in \mathbb{R}^{n}\right\} .
\end{aligned}
$$

If $(2.3)$ holds for a special case when $s=m$ and $\lambda_{i}=2$ for $i=1,2, \ldots, m$, then $\mathcal{F}$ is called co-quadratic M-quasiconvex to be introduced below.

Definition 2.7. Suppose $F$ is a nonnegative form associated with a $2 m$ th order symmetric tensor $\mathcal{F} \in \mathbb{R}^{n^{2 m}}$. The tensor $\mathcal{F}$ is called co-quadratic M-quasiconvex if

$$
F\left(\boldsymbol{x}_{1}, \boldsymbol{x}_{1}, \boldsymbol{x}_{2}, \boldsymbol{x}_{2}, \ldots, \boldsymbol{x}_{m}, \boldsymbol{x}_{m}\right) \leq \max _{1 \leq i \leq m}\left\{F(\underbrace{\boldsymbol{x}_{i}, \boldsymbol{x}_{i}, \ldots, \boldsymbol{x}_{i}}_{2 m})\right\} \quad \forall \boldsymbol{x}_{1}, \boldsymbol{x}_{2}, \ldots, \boldsymbol{x}_{m} \in \mathbb{R}^{n} .
$$


We denote the set of co-quadratic M-quasiconvex tensors to be

$$
\begin{aligned}
\mathbb{M}_{2}^{n^{2 m}}:=\left\{\mathcal{F} \in \mathbb{S}_{+}^{n^{2 m}} \mid\right. & F\left(\boldsymbol{x}_{1}, \boldsymbol{x}_{1}, \boldsymbol{x}_{2}, \boldsymbol{x}_{2}, \ldots, \boldsymbol{x}_{m}, \boldsymbol{x}_{m}\right) \\
& \left.\leq \max _{1 \leq i \leq m}\left\{F(\underbrace{\boldsymbol{x}_{i}, \boldsymbol{x}_{i}, \ldots, \boldsymbol{x}_{i}}_{2 m})\right\} \forall \boldsymbol{x}_{1}, \boldsymbol{x}_{2}, \ldots, \boldsymbol{x}_{m} \in \mathbb{R}^{n}\right\} .
\end{aligned}
$$

Trivially we have $\mathbb{M}^{n^{2 m}} \subseteq \mathbb{M}_{2}^{n^{2 m}}$.

We remark that in Definition 2.6, (2.3) already implies that $\mathcal{F}$ is nonnegative. This is because if we let $\boldsymbol{x}_{1}=-\boldsymbol{x}$ and $\boldsymbol{x}_{i}=\boldsymbol{x}$ for $i=2,3, \ldots, 2 m$ in (2.4), then

$$
F(-\boldsymbol{x}, \underbrace{\boldsymbol{x}, \boldsymbol{x}, \ldots, \boldsymbol{x}}_{2 m-1}) \leq F(\underbrace{\boldsymbol{x}, \boldsymbol{x}, \ldots, \boldsymbol{x}}_{2 m}),
$$

implying the nonnegativity of $\mathcal{F}$. However, in Definition 2.7, (2.6) does not imply the nonnegativity of $\mathcal{F}$, as shown by the following example.

Example 2.8. Let $\mathcal{F}=-1 \in \mathbb{R}^{1^{4}}$ be a 4 th order symmetric tensor and its associated multilinear form $F(x, y, z, w)=-x y z w$ for $x, y, z, w \in \mathbb{R}$. We have

$$
F(x, x, y, y)=-x^{2} y^{2} \leq \max \left\{-x^{4},-y^{4}\right\}=\max \{F(x, x, x, x), F(y, y, y, y)\},
$$

implying (2.6). However $\mathcal{F}$ is clearly not nonnegative.

We also remark that $\mathcal{F}$ being nonnegative is important for our discussion. Without nonnegativity, the cone consisting of all co-quadratic M-quasiconvex tensors in $\mathbb{S}^{n^{2 m}}$ is not even convex; see Example 2.9. However, with nonnegativity this cone is convex as Corollary 3.4 will stipulate. The nonnegativity of $\mathcal{F}$ also ensures that the right-hand sides of (2.3) and (2.6) will always be nonnegative. For consistency of expression we still keep the requirement of nonnegativity in Definition 2.6 although it is actually redundant in that case. Throughout this paper, the cone of nonnegative symmetric tensors (or the cone of nonnegative forms) is the ground set for our discussion.

Example 2.9. Let $\mathcal{F}_{1}, \mathcal{F}_{2} \in \mathbb{R}^{2^{4}}$ be 4 th order symmetric tensors, with their associated multilinear forms being $F_{1}(\boldsymbol{x}, \boldsymbol{y}, \boldsymbol{z}, \boldsymbol{w})=-2 x_{1} y_{1} z_{1} w_{1}$ and $F_{2}(\boldsymbol{x}, \boldsymbol{y}, \boldsymbol{z}, \boldsymbol{w})=$ $-2 x_{2} y_{2} z_{2} w_{2}$. According to Example 2.8, both $F_{1}$ and $F_{2}$ satisfy (2.6). However $F(\boldsymbol{x}, \boldsymbol{y}, \boldsymbol{z}, \boldsymbol{w})=-x_{1} y_{1} z_{1} w_{1}-x_{2} y_{2} z_{2} w_{2}$ with its associated tensor $\mathcal{F}=\frac{1}{2}\left(\mathcal{F}_{1}+\mathcal{F}_{2}\right)$ does not satisfy $(2.6)$, since

$$
\begin{aligned}
F(\boldsymbol{x}, \boldsymbol{x}, \boldsymbol{y}, \boldsymbol{y}) & =-x_{1}^{2} y_{1}^{2}-x_{2}^{2} y_{2}^{2} \\
& >\max \left\{-x_{1}^{4}-x_{2}^{4},-y_{1}^{4}-y_{2}^{4}\right\} \\
& =\max \{F(\boldsymbol{x}, \boldsymbol{x}, \boldsymbol{x}, \boldsymbol{x}), F(\boldsymbol{y}, \boldsymbol{y}, \boldsymbol{y}, \boldsymbol{y})\},
\end{aligned}
$$

when $\boldsymbol{x}=(1,2)^{\mathrm{T}}$ and $\boldsymbol{y}=(2,1)^{\mathrm{T}}$.

To simplify the notation, whenever appropriate we now use superscripts to simplify the form

$$
F(\underbrace{\boldsymbol{x}_{1}, \ldots, \boldsymbol{x}_{1}}_{\lambda_{1}}, \underbrace{\boldsymbol{x}_{2}, \ldots, \boldsymbol{x}_{2}}_{\lambda_{2}}, \ldots, \underbrace{\boldsymbol{x}_{s}, \ldots, \boldsymbol{x}_{s}}_{\lambda_{s}})
$$


i.e., $F\left(\boldsymbol{x}_{1}^{\lambda_{1}} \boldsymbol{x}_{2}^{\lambda_{2}} \ldots \boldsymbol{x}_{s}^{\lambda_{s}}\right)$. For example, $F\left(\boldsymbol{x}^{2} \boldsymbol{y}^{2}\right)$ denotes $F(\boldsymbol{x}, \boldsymbol{x}, \boldsymbol{y}, \boldsymbol{y})$, which is equal to $F(\boldsymbol{x}, \boldsymbol{y}, \boldsymbol{x}, \boldsymbol{y})$ (or any possible permutation of the positions of the entry vectors due to the symmetry). With this simplified notation, (2.3) is then

$$
F\left(\boldsymbol{x}_{1}^{\lambda_{1}} \boldsymbol{x}_{2}^{\lambda_{2}} \ldots \boldsymbol{x}_{s}^{\lambda_{s}}\right) \leq \max _{1 \leq i \leq s}\left\{F\left(\boldsymbol{x}_{i}^{2 m}\right)\right\} .
$$

In the same spirit, the homogeneous polynomial $f(\boldsymbol{x})$ associated with the multilinear form $F$ or the symmetric tensor $\mathcal{F}$ can be simply written as $F\left(\boldsymbol{x}^{2 m}\right)$.

Remark that Definitions 2.1, 2.2, 2.6, and 2.7 are all difficult to check in general. However, as we will discuss later, in specific applications it is often possible to find useful functions that satisfy these conditions. The situation is like verifying convexity in general might be difficult according to its definition; however, there are sufficient verifiable conditions assuring the property (e.g., a quadratic form with positive semidefinite Hessian). Finally, we conclude this section by pointing out that Definitions $2.1,2.2,2.6$, and 2.7 are actually identical in the matrix setting, i.e., when $m=1$ we have $\mathbb{S}_{+}^{n^{2}}=\mathbb{S}_{2+}^{n^{2}}=\mathbb{M}^{n^{2}}=\mathbb{M}_{2}^{n^{2}}$.

Proposition 2.10. If $\boldsymbol{x}, \boldsymbol{y} \in \mathbb{R}^{n}$ and symmetric matrix $Q \in \mathbb{R}^{n \times n}$ is positive semidefinite, then the bilinear form $F(\boldsymbol{x}, \boldsymbol{y})=\boldsymbol{x}^{\mathrm{T}} Q \boldsymbol{y}$ is M-quasiconvex. Conversely, if $\boldsymbol{x}^{\mathrm{T}} Q \boldsymbol{y}$ is M-quasiconvex, then $Q$ is positive semidefinite.

Proof. For all $\boldsymbol{x}, \boldsymbol{y} \in \mathbb{R}^{n}, Q$ being positive semidefinite implies $(\boldsymbol{x}-\boldsymbol{y})^{\mathrm{T}} Q(\boldsymbol{x}-\boldsymbol{y}) \geq$ 0 , which is $2 \boldsymbol{x}^{\mathrm{T}} Q \boldsymbol{y} \leq \boldsymbol{x}^{\mathrm{T}} Q \boldsymbol{x}+\boldsymbol{y}^{\mathrm{T}} Q \boldsymbol{y}$. This further leads to

$$
\boldsymbol{x}^{\mathrm{T}} Q \boldsymbol{y} \leq \max \left\{\boldsymbol{x}^{\mathrm{T}} Q \boldsymbol{x}, \boldsymbol{y}^{\mathrm{T}} Q \boldsymbol{y}\right\}
$$

implying that $F$ is M-quasiconvex. Conversely, M-quasiconvexity of $F$ implies that

$$
F(-\boldsymbol{x}, \boldsymbol{x}) \leq \max \{F(-\boldsymbol{x},-\boldsymbol{x}), F(\boldsymbol{x}, \boldsymbol{x})\}=F(\boldsymbol{x}, \boldsymbol{x}) .
$$

Therefore $\boldsymbol{x}^{\mathrm{T}} Q \boldsymbol{x}=F(\boldsymbol{x}, \boldsymbol{x}) \geq 0$, implying that $Q$ is positive semidefinite.

3. Equivalent definitions. In this section we present a curious fact that the newly introduced M-quasiconvexity is actually equivalent to some of the seemingly more restrictive definitions.

Lemma 3.1. Suppose $F$ is a nonnegative form associated with a $2 m$ th order symmetric tensor $\mathcal{F} \in \mathbb{S}^{n^{2 m}}$. The following three statements are equivalent:

$$
\begin{array}{ll}
F\left(\boldsymbol{x}_{1} \boldsymbol{x}_{2} \ldots \boldsymbol{x}_{2 m}\right) \leq \max _{1 \leq i \leq 2 m}\left\{F\left(\boldsymbol{x}_{i}^{2 m}\right)\right\} & \forall \boldsymbol{x}_{1}, \boldsymbol{x}_{2}, \ldots, \boldsymbol{x}_{2 m} \in \mathbb{R}^{n}, \\
F\left(\boldsymbol{x}_{1} \boldsymbol{x}_{2} \ldots \boldsymbol{x}_{2 m}\right) \leq \frac{1}{2 m} \sum_{i=1}^{2 m} F\left(\boldsymbol{x}_{i}^{2 m}\right) \quad \forall \boldsymbol{x}_{1}, \boldsymbol{x}_{2}, \ldots, \boldsymbol{x}_{2 m} \in \mathbb{R}^{n}, \\
F\left(\boldsymbol{x}_{1} \boldsymbol{x}_{2} \ldots \boldsymbol{x}_{2 m}\right) \leq\left(\prod_{i=1}^{2 m} F\left(\boldsymbol{x}_{i}^{2 m}\right)\right)^{\frac{1}{2 m}} \quad \forall \boldsymbol{x}_{1}, \boldsymbol{x}_{2}, \ldots, \boldsymbol{x}_{2 m} \in \mathbb{R}^{n} .
\end{array}
$$

Proof. Since $F\left(\boldsymbol{x}_{i}^{2 m}\right) \geq 0$ for all $\boldsymbol{x}_{i} \in \mathbb{R}^{n}$, it follows immediately by the mean inequalities that $(3.3) \Rightarrow(3.2) \Rightarrow(3.1)$. It remains to prove that $(3.1) \Rightarrow(3.3)$.

For any $a_{1}, a_{2}, \ldots, a_{2 m} \in \mathbb{R}$ and $\boldsymbol{x}_{1}, \boldsymbol{x}_{2}, \ldots, \boldsymbol{x}_{2 m} \in \mathbb{R}^{n}$, by the multilinearity of $F$, it follows from (3.1) that 


$$
\begin{aligned}
a_{1} a_{2} \ldots a_{2 m} F\left(\boldsymbol{x}_{1} \boldsymbol{x}_{2} \ldots \boldsymbol{x}_{2 m}\right) & =F\left(a_{1} \boldsymbol{x}_{1}, a_{2} \boldsymbol{x}_{2}, \ldots, a_{2 m} \boldsymbol{x}_{2 m}\right) \\
& \leq \max _{1 \leq i \leq 2 m}\left\{F\left(\left(a_{i} \boldsymbol{x}_{i}\right)^{2 m}\right)\right\} \\
& =\max _{1 \leq i \leq 2 m}\left\{a_{i}^{2 m} F\left(\boldsymbol{x}_{i}^{2 m}\right)\right\} .
\end{aligned}
$$

If $F\left(\boldsymbol{x}_{i}^{2 m}\right)>0$ for all $i=1,2, \ldots, 2 m$, then we choose $a_{i}>0$ such that $a_{i}^{2 m} F\left(\boldsymbol{x}_{i}^{2 m}\right)=1$ for all $i=1,2, \ldots, 2 m$. In that case, (3.4) leads to

$$
\left(\prod_{i=1}^{2 m}\left(F\left(\boldsymbol{x}_{i}^{2 m}\right)\right)^{-\frac{1}{2 m}}\right) F\left(\boldsymbol{x}_{1} \boldsymbol{x}_{2} \ldots \boldsymbol{x}_{2 m}\right) \leq 1,
$$

which implies (3.3).

Otherwise, there exists some $1 \leq i \leq 2 m$ satisfying $F\left(\boldsymbol{x}_{i}^{2 m}\right)=0$. Without loss of generality we assume $F\left(\boldsymbol{x}_{1}^{2 m}\right)=0$. Letting $a_{2}=a_{3}=\cdots=a_{2 m}=1$ in (3.4) leads to

$$
a_{1} F\left(\boldsymbol{x}_{1} \boldsymbol{x}_{2} \ldots \boldsymbol{x}_{2 m}\right) \leq \max \left\{\max _{2 \leq i \leq 2 m}\left\{F\left(\boldsymbol{x}_{i}^{2 m}\right)\right\}, a_{1}^{2 m} F\left(\boldsymbol{x}_{1}^{2 m}\right)\right\}=\max _{2 \leq i \leq 2 m}\left\{F\left(\boldsymbol{x}_{i}^{2 m}\right)\right\}
$$

for all $a_{1} \in \mathbb{R}$. The above clearly implies that $F\left(\boldsymbol{x}_{1} \boldsymbol{x}_{2} \ldots \boldsymbol{x}_{2 m}\right)=0$. Therefore (3.3) is satisfied as both sides are now zeros.

In fact, the above equivalence indicates that any generalized mean with exponent $p \in(0,+\infty]$ of $F\left(\boldsymbol{x}_{1}^{2 m}\right), F\left(\boldsymbol{x}_{2}^{2 m}\right), \ldots, F\left(\boldsymbol{x}_{2 m}^{2 m}\right)$ on the right-hand side can serve as the definition for M-quasiconvexity. Specifically, a symmetric tensor $\mathcal{F}$ is M-quasiconvex if and only if

$$
F\left(\boldsymbol{x}_{1} \boldsymbol{x}_{2} \ldots \boldsymbol{x}_{2 m}\right) \leq\left(\frac{1}{2 m} \sum_{i=1}^{2 m}\left(F\left(\boldsymbol{x}_{i}^{2 m}\right)\right)^{p}\right)^{\frac{1}{p}} \quad \forall \boldsymbol{x}_{1}, \boldsymbol{x}_{2}, \ldots, \boldsymbol{x}_{2 m} \in \mathbb{R}^{n}
$$

for any fixed $p \in(0,+\infty]$. By Lemma 3.1, we may equivalently write

$$
\mathbb{M}^{n^{2 m}}=\left\{\mathcal{F} \in \mathbb{S}_{+}^{n^{2 m}} \mid F\left(\boldsymbol{x}_{1} \boldsymbol{x}_{2} \ldots \boldsymbol{x}_{2 m}\right) \leq \frac{1}{2 m} \sum_{i=1}^{2 m} F\left(\boldsymbol{x}_{i}^{2 m}\right) \forall \boldsymbol{x}_{1}, \boldsymbol{x}_{2}, \ldots, \boldsymbol{x}_{2 m} \in \mathbb{R}^{n}\right\} .
$$

This formulation essentially implies the convexity of the set $\mathbb{M}^{n^{2 m}}$, which does not follow straightforwardly from its original definition (2.5). Therefore we have the following.

Corollary 3.2. The set of all M-quasiconvex tensors (i.e., $\mathbb{M}^{n^{2 m}}$ ) is a closed convex cone.

Similarly we have the following equivalent definitions for co-quadratic M-quasiconvex tensors, whose proof is similar and is omitted here.

Lemma 3.3. Suppose $F$ is a nonnegative form associated with a $2 \mathrm{~m}$ th order symmetric tensor $\mathcal{F} \in \mathbb{S}^{n^{2 m}}$. The following three statements are equivalent:

$$
\begin{aligned}
& F\left(\boldsymbol{x}_{1}^{2} \boldsymbol{x}_{2}^{2} \ldots \boldsymbol{x}_{m}^{2}\right) \leq \max _{1 \leq i \leq m}\left\{F\left(\boldsymbol{x}_{i}^{2 m}\right)\right\} \quad \forall \boldsymbol{x}_{1}, \boldsymbol{x}_{2}, \ldots, \boldsymbol{x}_{m} \in \mathbb{R}^{n}, \\
& F\left(\boldsymbol{x}_{1}^{2} \boldsymbol{x}_{2}^{2} \ldots \boldsymbol{x}_{m}^{2}\right) \leq \frac{1}{m} \sum_{i=1}^{m} F\left(\boldsymbol{x}_{i}^{2 m}\right) \quad \forall \boldsymbol{x}_{1}, \boldsymbol{x}_{2}, \ldots, \boldsymbol{x}_{m} \in \mathbb{R}^{n}, \\
& F\left(\boldsymbol{x}_{1}^{2} \boldsymbol{x}_{2}^{2} \ldots \boldsymbol{x}_{m}^{2}\right) \leq\left(\prod_{i=1}^{m} F\left(\boldsymbol{x}_{i}^{2 m}\right)\right)^{\frac{1}{m}} \quad \forall \boldsymbol{x}_{1}, \boldsymbol{x}_{2}, \ldots, \boldsymbol{x}_{m} \in \mathbb{R}^{n} .
\end{aligned}
$$


We also have

$$
\mathbb{M}_{2}^{n^{2 m}}=\left\{\mathcal{F} \in \mathbb{S}_{+}^{n^{2 m}} \mid F\left(\boldsymbol{x}_{1}^{2} \boldsymbol{x}_{2}^{2} \ldots \boldsymbol{x}_{m}^{2}\right) \leq \frac{1}{m} \sum_{i=1}^{m} F\left(\boldsymbol{x}_{i}^{2 m}\right) \forall \boldsymbol{x}_{1}, \boldsymbol{x}_{2}, \ldots, \boldsymbol{x}_{m} \in \mathbb{R}^{n}\right\},
$$

and consequently we have the following.

COROLlary 3.4. The set of all co-quadratic M-quasiconvex tensors (i.e., $\mathbb{M}_{2}^{n^{2 m}}$ ) is a closed convex cone.

4. The relationships. As mentioned at the end of section 2 , when the degree of the form is 2, then M-quasiconvex tensors, co-quadratic M-quasiconvex tensors and co-quadratic nonnegative tensors are all the same, i.e., $\mathbb{M}^{n^{2}}=\mathbb{M}_{2}^{n^{2}}=\mathbb{S}_{2+}^{n^{2}}$. In this section we establish the relationship for general degree $2 m$. Before presenting the main results, let us first study an important property for the co-quadratic nonnegative tensors.

LEMma 4.1. If $F$ is a co-quadratic nonnegative form associated with a $2 m$ th order symmetric tensor $\mathcal{F} \in \mathbb{S}^{n^{2 m}}$, then

$$
F\left(\boldsymbol{y}^{2 m}\right)+F\left(\boldsymbol{z}^{2 m}\right) \geq F\left(\boldsymbol{y}^{2 m-2} \boldsymbol{z}^{2}\right)+F\left(\boldsymbol{y}^{2} \boldsymbol{z}^{2 m-2}\right) \quad \forall \boldsymbol{y}, \boldsymbol{z} \in \mathbb{R}^{n} .
$$

Proof. The proof is based on induction on $m$. Obviously (4.1) holds when $m=1$. For the case $m=2$, due to the co-quadratic nonnegativity of $\mathcal{F}$, we have

$$
F(\boldsymbol{y}+\boldsymbol{z}, \boldsymbol{y}+\boldsymbol{z}, \boldsymbol{y}-\boldsymbol{z}, \boldsymbol{y}-\boldsymbol{z}) \geq 0 \quad \forall \boldsymbol{y}, \boldsymbol{z} \in \mathbb{R}^{n}
$$

that is,

$$
F\left(\boldsymbol{y}^{4}\right)+F\left(\boldsymbol{z}^{4}\right) \geq 2 F\left(\boldsymbol{y}^{2} \boldsymbol{z}^{2}\right) \quad \forall \boldsymbol{y}, \boldsymbol{z} \in \mathbb{R}^{n},
$$

which implies that (4.1) holds when $m=2$.

Suppose that (4.1) holds for tensors of order no more than $2 m$. For the sake of induction, we next consider tensors of order $2(m+1)$, and we wish to show the following inequality,

$$
F\left(\boldsymbol{y}^{2 m+2}\right)+F\left(\boldsymbol{z}^{2 m+2}\right) \geq F\left(\boldsymbol{y}^{2 m} \boldsymbol{z}^{2}\right)+F\left(\boldsymbol{y}^{2} \boldsymbol{z}^{2 m}\right) \quad \forall \boldsymbol{y}, \boldsymbol{z} \in \mathbb{R}^{n} .
$$

For any fixed $\boldsymbol{x} \in \mathbb{R}^{n}$ and $k=1,2, \ldots, m$, we define the following multilinear form

$$
G_{\boldsymbol{x}^{2 k}}\left(\boldsymbol{y}_{1} \boldsymbol{y}_{2} \ldots \boldsymbol{y}_{2 m+2-2 k}\right):=F\left(\boldsymbol{x}^{2 k} \boldsymbol{y}_{1} \boldsymbol{y}_{2} \ldots \boldsymbol{y}_{2 m+2-2 k}\right) \text {. }
$$

By the co-quadratic nonnegativity of $F, G_{\boldsymbol{x}^{2 k}}$ is a co-quadratic nonnegative form associated with a $(2 m+2-2 k)$ th order symmetric tensor. Therefore, by the induction assumption, we have for $k=1,2, \ldots, m$,

$$
G_{\boldsymbol{x}^{2 k}}\left(\boldsymbol{y}^{2 m+2-2 k}\right)+G_{\boldsymbol{x}^{2 k}}\left(\boldsymbol{z}^{2 m+2-2 k}\right) \geq G_{\boldsymbol{x}^{2 k}}\left(\boldsymbol{y}^{2 m-2 k} \boldsymbol{z}^{2}\right)+G_{\boldsymbol{x}^{2 k}}\left(\boldsymbol{y}^{2} \boldsymbol{z}^{2 m-2 k}\right),
$$

i.e.,

$$
F\left(\boldsymbol{x}^{2 k} \boldsymbol{y}^{2 m+2-2 k}\right)+F\left(\boldsymbol{x}^{2 k} \boldsymbol{z}^{2 m+2-2 k}\right) \geq F\left(\boldsymbol{x}^{2 k} \boldsymbol{y}^{2 m-2 k} \boldsymbol{z}^{2}\right)+F\left(\boldsymbol{x}^{2 k} \boldsymbol{y}^{2} \boldsymbol{z}^{2 m-2 k}\right) .
$$

Summing over $k=1,2, \ldots, m$ in the above inequality, and letting $\boldsymbol{x}=\boldsymbol{y}$ and $\boldsymbol{x}=\boldsymbol{z}$, respectively, we have 


$$
\begin{aligned}
& \sum_{k=1}^{m}\left(F\left(\boldsymbol{y}^{2 m+2}\right)+F\left(\boldsymbol{y}^{2 k} \boldsymbol{z}^{2 m+2-2 k}\right)\right) \geq \sum_{k=1}^{m}\left(F\left(\boldsymbol{y}^{2 m} \boldsymbol{z}^{2}\right)+F\left(\boldsymbol{y}^{2 k+2} \boldsymbol{z}^{2 m-2 k}\right)\right) \\
& \sum_{k=1}^{m}\left(F\left(\boldsymbol{y}^{2 m+2-2 k} \boldsymbol{z}^{2 k}\right)+F\left(\boldsymbol{z}^{2 m+2}\right)\right) \geq \sum_{k=1}^{m}\left(F\left(\boldsymbol{y}^{2 m-2 k} \boldsymbol{z}^{2 k+2}\right)+F\left(\boldsymbol{y}^{2} \boldsymbol{z}^{2 m}\right)\right) .
\end{aligned}
$$

Adding up these two inequalities and canceling out the same terms on both sides leads to

$$
(m-1)\left(F\left(\boldsymbol{y}^{2 m+2}\right)+F\left(\boldsymbol{z}^{2 m+2}\right)\right) \geq(m-1)\left(F\left(\boldsymbol{y}^{2 m} \boldsymbol{z}^{2}\right)+F\left(\boldsymbol{y}^{2} \boldsymbol{z}^{2 m}\right)\right),
$$

which establishes the inductive step (4.2).

Our first main result says that co-quadratic nonnegativity implies co-quadratic M-quasiconvexity.

THEOREM 4.2. If $F$ is a co-quadratic nonnegative form associated with an even order symmetric tensor $\mathcal{F} \in \mathbb{S}^{2 m}$, then it is also co-quadratic M-quasiconvex, i.e.,

$$
F\left(\boldsymbol{y}_{1}^{2} \boldsymbol{y}_{2}^{2} \ldots \boldsymbol{y}_{m}^{2}\right) \geq 0 \forall \boldsymbol{y}_{i} \in \mathbb{R}^{n} \Longrightarrow F\left(\boldsymbol{x}_{1}^{2} \boldsymbol{x}_{2}^{2} \ldots \boldsymbol{x}_{m}^{2}\right) \leq \max _{1 \leq i \leq m}\left\{F\left(\boldsymbol{x}_{i}^{2 m}\right)\right\} \forall \boldsymbol{x}_{i} \in \mathbb{R}^{n}
$$

In other words, we have $\mathbb{S}_{2+}^{n^{2 m}} \subseteq \mathbb{M}_{2}^{n^{2 m}}$.

Proof. We prove the co-quadratic M-quasiconvexity using (3.5), i.e.,

$$
\sum_{i=1}^{m} F\left(\boldsymbol{x}_{i}^{2 m}\right) \geq m F\left(\boldsymbol{x}_{1}^{2} \boldsymbol{x}_{2}^{2} \ldots \boldsymbol{x}_{m}^{2}\right) \quad \forall \boldsymbol{x}_{1}, \boldsymbol{x}_{2}, \ldots, \boldsymbol{x}_{m} \in \mathbb{R}^{n}
$$

by induction on $m$. It is trivially true when $m=1$. If it holds for the case $m$, then for the case $m+1$, we need to show that

$$
\sum_{i=1}^{m+1} F\left(\boldsymbol{x}_{i}^{2 m+2}\right) \geq(m+1) F\left(\boldsymbol{x}_{1}^{2} \boldsymbol{x}_{2}^{2} \ldots \boldsymbol{x}_{m}^{2} \boldsymbol{x}_{m+1}^{2}\right) \quad \forall \boldsymbol{x}_{1}, \boldsymbol{x}_{2}, \ldots, \boldsymbol{x}_{m}, \boldsymbol{x}_{m+1} \in \mathbb{R}^{n}
$$

According to Lemma 4.1 we have

$$
F\left(\boldsymbol{x}_{i}^{2 m+2}\right)+F\left(\boldsymbol{x}_{j}^{2 m+2}\right) \geq F\left(\boldsymbol{x}_{i}^{2} \boldsymbol{x}_{j}^{2 m}\right)+F\left(\boldsymbol{x}_{i}^{2 m} \boldsymbol{x}_{j}^{2}\right) \quad \forall 1 \leq i, j \leq m+1 .
$$

Summing over all $i<j$ further leads to

$$
\sum_{1 \leq i<j \leq m+1}\left(F\left(\boldsymbol{x}_{i}^{2 m+2}\right)+F\left(\boldsymbol{x}_{j}^{2 m+2}\right)\right) \geq \sum_{1 \leq i<j \leq m+1}\left(F\left(\boldsymbol{x}_{i}^{2} \boldsymbol{x}_{j}^{2 m}\right)+F\left(\boldsymbol{x}_{i}^{2 m} \boldsymbol{x}_{j}^{2}\right)\right),
$$

which implies that

$$
m \sum_{i=1}^{m+1} F\left(\boldsymbol{x}_{i}^{2 m+2}\right) \geq \sum_{i=1}^{m+1} \sum_{j \neq i} F\left(\boldsymbol{x}_{i}^{2} \boldsymbol{x}_{j}^{2 m}\right) \geq \sum_{i=1}^{m+1} m F\left(\boldsymbol{x}_{i}^{2} \prod_{j \neq i} \boldsymbol{x}_{j}^{2}\right)=m(m+1) F\left(\prod_{j=1}^{m+1} \boldsymbol{x}_{j}^{2}\right)
$$


where in the second inequality, the induction assumption on $m$ is applied, since for any $\boldsymbol{x}_{i} \in \mathbb{R}^{n}$, the multilinear form $G_{\boldsymbol{x}_{i}}$ satisfies

$$
G_{\boldsymbol{x}_{i}}\left(\boldsymbol{y}_{1}^{2} \boldsymbol{y}_{2}^{2} \ldots \boldsymbol{y}_{m}^{2}\right):=F\left(\boldsymbol{x}_{i}^{2} \boldsymbol{y}_{1}^{2} \boldsymbol{y}_{2}^{2} \ldots \boldsymbol{y}_{m}^{2}\right) \geq 0 \quad \forall \boldsymbol{y}_{1}, \boldsymbol{y}_{2}, \ldots, \boldsymbol{y}_{m} \in \mathbb{R}^{n}
$$

and is therefore co-quadratic nonnegative. This completes the induction step (4.3), which concludes the whole proof.

As co-quadratic M-quasiconvexity is more general than M-quasiconvexity, here we provide a stronger statement.

THEOREM 4.3. If $F$ is a co-quadratic nonnegative form associated with a $2 \mathrm{mth}$ order symmetric tensor $\mathcal{F} \in \mathbb{S}^{n^{2 m}}$, then it is also M-quasiconvex, i.e.,

$$
F\left(\boldsymbol{y}_{1}^{2} \boldsymbol{y}_{2}^{2} \ldots \boldsymbol{y}_{m}^{2}\right) \geq 0 \forall \boldsymbol{y}_{i} \in \mathbb{R}^{n} \Longrightarrow F\left(\boldsymbol{x}_{1} \boldsymbol{x}_{2} \ldots \boldsymbol{x}_{2 m}\right) \leq \max _{1 \leq i \leq 2 m}\left\{F\left(\boldsymbol{x}_{i}^{2 m}\right)\right\} \forall \boldsymbol{x}_{i} \in \mathbb{R}^{n} .
$$

In other words, $\mathbb{S}_{2+}^{n^{2 m}} \subseteq \mathbb{M}^{n^{2 m}}$.

Proof. Let $\xi_{1}, \xi_{2}, \ldots, \xi_{m}$ be Bernoulli random variables, each taking values 1 and -1 with equal probability, satisfying $\prod_{i=1}^{m} \xi_{i}=-1$. For any $\boldsymbol{x}_{1}, \boldsymbol{x}_{2}, \ldots, \boldsymbol{x}_{2 m} \in \mathbb{R}^{n}$, since $F$ is co-quadratic nonnegative, we observe that

$$
\begin{aligned}
0 & \leq \mathrm{E}\left[F\left(\left(\boldsymbol{x}_{1}+\xi_{1} \boldsymbol{x}_{2}\right)^{2}\left(\boldsymbol{x}_{3}+\xi_{2} \boldsymbol{x}_{4}\right)^{2} \ldots\left(\boldsymbol{x}_{2 m-1}+\xi_{m} \boldsymbol{x}_{2 m}\right)^{2}\right)\right] \\
& =\sum_{i_{1}=1}^{2} \sum_{i_{2}=3}^{4} \ldots \sum_{i_{m}=2 m-1}^{2 m} F\left(\boldsymbol{x}_{i_{1}}^{2} \boldsymbol{x}_{i_{2}}^{2} \ldots \boldsymbol{x}_{i_{m}}^{2}\right)-2^{m} F\left(\boldsymbol{x}_{1} \boldsymbol{x}_{2} \ldots \boldsymbol{x}_{2 m}\right) .
\end{aligned}
$$

This is because the function $F$ is multilinear, and the expectation of the coefficient for any term other than those in the right-hand side of (4.4) is zero.

Since $F$ is co-quadratic nonnegative, by Theorem 4.2 it is co-quadratic M-quasiconvex, and we have

$$
F\left(\boldsymbol{x}_{i_{1}}^{2} \boldsymbol{x}_{i_{2}}^{2} \ldots \boldsymbol{x}_{i_{m}}^{2}\right) \leq \max \left\{F\left(\boldsymbol{x}_{i_{1}}^{2 m}\right), F\left(\boldsymbol{x}_{i_{2}}^{2 m}\right), \ldots, F\left(\boldsymbol{x}_{i_{m}}^{2 m}\right)\right\} \leq \max _{1 \leq i \leq 2 m}\left\{F\left(\boldsymbol{x}_{i}^{2 m}\right)\right\}
$$

for all $i_{1} \in\{1,2\}, i_{2} \in\{3,4\}, \ldots, i_{m} \in\{2 m-1,2 m\}$. Therefore (4.4) further leads to

$$
2^{m} F\left(\boldsymbol{x}_{1} \boldsymbol{x}_{2} \ldots \boldsymbol{x}_{2 m}\right) \leq \sum_{i_{1}=1}^{2} \sum_{i_{2}=3}^{4} \ldots \sum_{i_{m}=2 m-1}^{2 m} F\left(\boldsymbol{x}_{i_{1}}^{2} \boldsymbol{x}_{i_{2}}^{2} \ldots \boldsymbol{x}_{i_{m}}^{2}\right) \leq 2^{m} \max _{1 \leq i \leq 2 m}\left\{F\left(\boldsymbol{x}_{i}^{2 m}\right)\right\},
$$

proving that $F$ is M-quasiconvex.

To summarize, we provide the following result, which is an immediate consequence of Theorem 4.3 and Definitions 2.6 and 2.7.

Corollary 4.4. A co-quadratic nonnegative tensor is also M-quasiconvex, and an M-quasiconvex tensor is also co-quadratic M-quasiconvex, i.e.,

$$
\mathbb{S}_{2+}^{n^{2 m}} \subseteq \mathbb{M}^{n^{2 m}} \subseteq \mathbb{M}_{2}^{n^{2 m}}
$$

A natural question arises regarding the membership queries of these new cones of nonnegative tensors. As we shall see in the next section, Theorem 5.1 tells us that it is NP-hard to check the M-quasiconvexity or co-quadratic M-quasiconvexity for a quartic form. For higher orders, we believe these classes of nonnegative tensors are NP-hard to verify. However, there is a tractable sufficient condition for these nonnegative tensors. 
THEOREM 4.5. Let $\mathcal{F} \in \mathbb{S}^{n^{2 m}}$ and rewrite it as an $n^{m} \times n^{m}$ symmetric matrix $M(\mathcal{F})$ by combining its $m$ modes into one mode (the row of $M(\mathcal{F})$ ) and the other $m$ modes into the other mode (the column of $M(\mathcal{F})$ ). If $M(\mathcal{F})$ is positive semidefinite, then $\mathcal{F}$ is co-quadratic nonnegative (hence $M$-quasiconvex or co-quadratic M-quasiconvex).

Proof. Since $\mathcal{F}$ is symmetric, it is easy to verify that

$$
\begin{aligned}
F & \left(\boldsymbol{x}_{1}, \boldsymbol{x}_{1}, \boldsymbol{x}_{2}, \boldsymbol{x}_{2}, \ldots, \boldsymbol{x}_{m}, \boldsymbol{x}_{m}\right) \\
& =F\left(\boldsymbol{x}_{1}, \boldsymbol{x}_{2}, \ldots, \boldsymbol{x}_{m}, \boldsymbol{x}_{1}, \boldsymbol{x}_{2}, \ldots, \boldsymbol{x}_{m}\right) \\
& =\left\langle\mathcal{F}, \boldsymbol{x}_{1} \circ \boldsymbol{x}_{2} \circ \cdots \circ \boldsymbol{x}_{m} \circ \boldsymbol{x}_{1} \circ \boldsymbol{x}_{2} \circ \cdots \circ \boldsymbol{x}_{m}\right\rangle \\
& =\left(\operatorname{vec}\left(\boldsymbol{x}_{1} \circ \boldsymbol{x}_{2} \circ \cdots \circ \boldsymbol{x}_{m}\right)\right)^{\mathrm{T}} M(\mathcal{F}) \operatorname{vec}\left(\boldsymbol{x}_{1} \circ \boldsymbol{x}_{2} \circ \cdots \circ \boldsymbol{x}_{m}\right) \\
& \geq 0,
\end{aligned}
$$

where the last inequality is due to positive semidefiniteness of $M(\mathcal{F})$. Therefore $\mathcal{F}$ is co-quadratic nonnegative.

Notice that verifying the positive semidefiniteness of $M(\mathcal{F})$ can be done easily, and runs in polynomial time. In the literature, such a symmetric tensor $\mathcal{F}$ is known as a matrix positive semidefinite tensor, proposed in [16] to study the cones of nonnegative quartic forms. A matrix positive semidefinite tensor is essentially equivalent to a positive semidefinite moment matrix (see, e.g., [22]). We also remark that although it is in general hard to check the M-quasiconvexity or co-quadratic M-quasiconvexity, it is essentially checking the nonnegativity of a (multivariate) polynomial function, which can be done in principle by resorting to some general purpose software tools such as GloptiPoly 3 [13] subject to the problem size (see Example 5.2).

5. Proper containments. The analysis in the previous section triggers the question about the further relationships among the sets $\mathbb{S}_{2+}^{n^{2 m}}, \mathbb{M}^{n^{2 m}}$, and $\mathbb{M}_{2}^{n^{2 m}}$. Our first result in this section generalizes the case $m=1$, which shows that these three sets are indeed the same even when $m=2$, i.e., $\mathbb{S}_{2+}^{n^{4}}=\mathbb{M}^{n^{4}}=\mathbb{M}_{2}^{n^{4}}$.

THEOREM 5.1. If $F$ is a nonnegative form associated with a 4 th order symmetric tensor $\mathcal{F} \in \mathbb{S}^{n^{4}}$, then the following three statements are equivalent:

1. $F$ is co-quadratic nonnegative, i.e., $F\left(\boldsymbol{x}_{1}^{2} \boldsymbol{x}_{2}^{2}\right) \geq 0 \forall \boldsymbol{x}_{1}, \boldsymbol{x}_{2} \in \mathbb{R}^{n}$.

2. $F$ is M-quasiconvex, i.e., $F\left(\boldsymbol{x}_{1} \boldsymbol{x}_{2} \boldsymbol{x}_{3} \boldsymbol{x}_{4}\right) \leq \max _{1 \leq i \leq 4}\left\{F\left(\boldsymbol{x}_{i}^{4}\right)\right\} \forall \boldsymbol{x}_{1}, \boldsymbol{x}_{2}, \boldsymbol{x}_{3}, \boldsymbol{x}_{4}$ $\in \mathbb{R}^{n}$.

3. $F$ is co-quadratic $M$-quasiconvex, i.e., $F\left(\boldsymbol{x}_{1}^{2} \boldsymbol{x}_{2}^{2}\right) \leq \max _{1 \leq i \leq 2}\left\{F\left(\boldsymbol{x}_{i}^{4}\right)\right\} \forall \boldsymbol{x}_{1}, \boldsymbol{x}_{2}$ $\in \mathbb{R}^{n}$

Proof. According to Corollary 4.4, we only need to show that co-quadratic M-quasiconvexity implies co-quadratic nonnegativity for a quartic form $F$, i.e., statement 3 implies statement 1 in Theorem 5.1. Indeed, by the equivalent property in Lemma $3.3, F$ is co-quadratic M-quasiconvex is equivalent to

$$
F\left(\boldsymbol{x}^{2} \boldsymbol{y}^{2}\right) \leq \frac{1}{2}\left(F\left(\boldsymbol{x}^{4}\right)+F\left(\boldsymbol{y}^{4}\right)\right) \quad \forall \boldsymbol{x}, \boldsymbol{y} \in \mathbb{R}^{n} .
$$

It leads to

$$
F\left((\boldsymbol{x}-\boldsymbol{y})^{2}(\boldsymbol{x}+\boldsymbol{y})^{2}\right)=F\left(\boldsymbol{x}^{4}\right)+F\left(\boldsymbol{y}^{4}\right)-2 F\left(\boldsymbol{x}^{2} \boldsymbol{y}^{2}\right) \geq 0 \quad \forall \boldsymbol{x}, \boldsymbol{y} \in \mathbb{R}^{n} .
$$

Therefore $F$ is co-quadratic nonnegative, completing the proof. 
As checking the convexity of a quartic form is in general NP-hard [1], Theorem 5.1 implies that checking the M-quasiconvexity or co-quadratic M-quasiconvexity for a quartic form is also NP-hard. Apart from the sufficient condition in Theorem 4.5, a weaker tractable sufficient condition known as the sos-convexity (cf. [12]) can also be useful for the quartic case, since sos-convexity implies the usual convexity. In general, the task of investigating the relationships among $\mathbb{S}_{2+}^{n^{2 m}}, \mathbb{M}^{n^{2 m}}$, and $\mathbb{M}_{2}^{n^{2 m}}$ for $m \geq 3$, however, becomes more complicated. Our second result asserts that at least two of these three cones are distinct when $m \geq 3$.

Example 5.2. Let $G\left(\boldsymbol{x}^{6}\right):=3 x_{1}^{6}+3 x_{2}^{6}+15 x_{1}^{4} x_{2}^{2}$ be a bivariate homogeneous polynomial associated with a 6 th order symmetric tensor $\mathcal{G} \in \mathbb{S}^{2}$, i.e., $\mathcal{G}_{111111}=\mathcal{G}_{222222}=$ $3, \mathcal{G}_{111122}=\mathcal{G}_{111212}=\cdots=1$, and all the rest of the entries are zeros. We have

1. $\mathcal{G}$ is nonnegative;

2. $\mathcal{G}$ is not co-quadratic nonnegative;

3. $G\left(\boldsymbol{x}^{2} \boldsymbol{y}^{4}\right) \geq 0 \forall \boldsymbol{x}, \boldsymbol{y} \in \mathbb{R}^{n}$;

4. $G\left(\boldsymbol{x}^{2} \boldsymbol{y}^{4}\right) \leq \max \left\{G\left(\boldsymbol{x}^{6}\right), G\left(\boldsymbol{y}^{6}\right)\right\} \forall \boldsymbol{x}, \boldsymbol{y} \in \mathbb{R}^{n}$;

5. $G\left(\boldsymbol{x}^{2} \boldsymbol{y}^{2} \boldsymbol{z}^{2}\right) \leq \max \left\{G\left(\boldsymbol{x}^{6}\right), G\left(\boldsymbol{y}^{6}\right), G\left(\boldsymbol{z}^{6}\right)\right\} \forall \boldsymbol{x}, \boldsymbol{y}, \boldsymbol{z} \in \mathbb{R}^{n}$ (G) is co-quadratic M-quasiconvex).

Toward proving these statements, we first notice that $G\left(\boldsymbol{x}^{6}\right)=3 x_{1}^{6}+3 x_{2}^{6}+$ $15 x_{1}^{4} x_{2}^{2} \geq 0$ for all $\boldsymbol{x} \in \mathbb{R}^{2}$, implying that $\mathcal{G}$ is nonnegative. Next by letting $\boldsymbol{x}=(15,1)^{\mathrm{T}}, \boldsymbol{y}=(-3,-13)^{\mathrm{T}}$, and $\boldsymbol{z}=(-5,10)^{\mathrm{T}}$ we have $G\left(\boldsymbol{x}^{2} \boldsymbol{y}^{2} \boldsymbol{z}^{2}\right)=-367575$, implying that $\mathcal{G}$ is not co-quadratic nonnegative.

For the third statement, we notice that for $\boldsymbol{x}, \boldsymbol{y} \in \mathbb{R}^{2}$

$$
\begin{aligned}
G\left(\boldsymbol{x}^{2} \boldsymbol{y}^{4}\right) & =3 x_{1}^{2} y_{1}^{4}+3 x_{2}^{2} y_{2}^{4}+x_{2}^{2} y_{1}^{4}+6 x_{1}^{2} y_{1}^{2} y_{2}^{2}+8 x_{1} x_{2} y_{1}^{3} y_{2} \\
& =\left(\begin{array}{l}
x_{1} \\
x_{2}
\end{array}\right)^{\mathrm{T}}\left[\begin{array}{cc}
3 y_{1}^{4}+6 y_{1}^{2} y_{2}^{2} & 4 y_{1}^{3} y_{2} \\
4 y_{1}^{3} y_{2} & y_{1}^{4}+3 y_{2}^{4}
\end{array}\right]\left(\begin{array}{l}
x_{1} \\
x_{2}
\end{array}\right) .
\end{aligned}
$$

Denote $Q=\left[\begin{array}{cc}3 y_{1}^{4}+6 y_{1}^{2} y_{2}^{2} & 4 y_{1}^{3} y_{2} \\ 4 y_{1}^{3} y_{2} & y_{1}^{4}+3 y_{2}^{4}\end{array}\right]$. By noticing that $Q_{11} \geq 0, Q_{22} \geq 0$ and

$$
\operatorname{det}(Q)=3 y_{1}^{8}+9 y_{1}^{4} y_{2}^{4}+18 y_{1}^{2} y_{2}^{6}-10 y_{1}^{6} y_{2}^{2}=\frac{\left(3 y_{1}^{4}-5 y_{1}^{2} y_{2}^{2}\right)^{2}}{3}+\frac{2 y_{1}^{4} y_{2}^{4}}{3}+18 y_{1}^{2} y_{2}^{6} \geq 0
$$

we have that $Q$ is positive semidefinite for any $\boldsymbol{y} \in \mathbb{R}^{2}$, proving the third statement. This is in fact equivalent to that $G\left(\boldsymbol{x}^{6}\right)$ is a convex function.

For the fourth statement, by Lemma 3.3 it suffices to show

$$
3 G\left(\boldsymbol{x}^{2} \boldsymbol{y}^{4}\right) \leq G\left(\boldsymbol{x}^{6}\right)+2 G\left(\boldsymbol{y}^{6}\right) \quad \forall \boldsymbol{x}, \boldsymbol{y} \in \mathbb{R}^{2} .
$$

Direct computation shows that

$$
\begin{aligned}
g_{1}(\boldsymbol{x}, \boldsymbol{y}):= & G\left(\boldsymbol{x}^{6}\right)+2 G\left(\boldsymbol{y}^{6}\right)-3 G\left(\boldsymbol{x}^{2} \boldsymbol{y}^{4}\right) \\
= & x_{1}^{6}+x_{2}^{6}+5 x_{1}^{4} x_{2}^{2}+2 y_{1}^{6}+2 y_{2}^{6}+10 y_{1}^{4} y_{2}^{2} \\
& -\left(3 x_{1}^{2} y_{1}^{4}+3 x_{2}^{2} y_{2}^{4}+x_{2}^{2} y_{1}^{4}+6 x_{1}^{2} y_{1}^{2} y_{2}^{2}+8 x_{1} x_{2} y_{1}^{3} y_{2}\right) .
\end{aligned}
$$

Establishing $g_{1}(\boldsymbol{x}, \boldsymbol{y}) \geq 0$ is not very easy. Instead, we shall prove this inequality by numerical optimization: we call GloptiPoly 3 [13] to solve the unconstrained polynomial optimization problem

$$
\min _{\boldsymbol{x}, \boldsymbol{y} \in \mathbb{R}^{2}} g_{1}(\boldsymbol{x}, \boldsymbol{y}) .
$$


When the relaxation order is set to be 3 , the global optimality is guaranteed for this problem, whose optimal value is zero with the optimal solution being $\boldsymbol{x}=\boldsymbol{y}=(0,0)^{\mathrm{T}}$. The fourth statement is thus verified.

For the last statement of Example 5.2, a similar method for the fourth statement is applied. By Lemma 3.3 we need to show that

$$
3 G\left(\boldsymbol{x}^{2} \boldsymbol{y}^{2} \boldsymbol{z}^{2}\right) \leq G\left(\boldsymbol{x}^{6}\right)+G\left(\boldsymbol{y}^{6}\right)+G\left(\boldsymbol{z}^{6}\right) \quad \forall \boldsymbol{x}, \boldsymbol{y}, \boldsymbol{z} \in \mathbb{R}^{2},
$$

which is

$$
\begin{aligned}
x_{1}^{6}+ & x_{2}^{6}+5 x_{1}^{4} x_{2}^{2}+y_{1}^{6}+y_{2}^{6}+5 y_{1}^{4} y_{2}^{2}+z_{1}^{6}+z_{2}^{6}+5 z_{1}^{4} z_{2}^{2}-\left(3 x_{1}^{2} y_{1}^{2} z_{1}^{2}+3 x_{2}^{2} y_{2}^{2} z_{2}^{2}\right. \\
& \left.+x_{1}^{2} y_{1}^{2} z_{2}^{2}+x_{1}^{2} y_{2}^{2} z_{1}^{2}+x_{2}^{2} y_{1}^{2} z_{1}^{2}+4 x_{1} x_{2} y_{1} y_{2} z_{1}^{2}+4 x_{1} x_{2} y_{1}^{2} z_{1} z_{2}+4 x_{1}^{2} y_{1} y_{2} z_{1} z_{2}\right) \\
= & : g_{2}(\boldsymbol{x}, \boldsymbol{y}, \boldsymbol{z}) \geq 0 .
\end{aligned}
$$

Applying Gloptipoly 3 to solve the problem

$$
\min _{\boldsymbol{x}, \boldsymbol{y}, \boldsymbol{z} \in \mathbb{R}^{2}} g_{2}(\boldsymbol{x}, \boldsymbol{y}, \boldsymbol{z}),
$$

and setting the relaxation order to be 4 , the global optimality is also certified. The optimal value for this problem is zero, with the optimal solution being $\boldsymbol{x}=\boldsymbol{y}=\boldsymbol{z}=$ $(0,0)^{\mathrm{T}}$. This proves the last statement, i.e., $\mathcal{G}$ is co-quadratic M-quasiconvex.

Example 5.2 clearly differentiates the co-quadratic nonnegativity and the co-quadratic M-quasiconvexity for sixth order symmetric tensors, which is not the case for quadratic and quartic tensors.

TheOREM 5.3. For $m \geq 3$ and $n \geq 2$, it holds that $\mathbb{S}_{2+}^{n^{2 m}} \subsetneq \mathbb{M}_{2}^{n^{2 m}}$.

One may certainly wonder about the status of $\mathbb{M}^{n^{2 m}}$ in the chain of containing relationship $\mathbb{S}_{2+}^{n^{2 m}} \subseteq \mathbb{M}^{2^{2 m}} \subseteq \mathbb{M}_{2}^{n^{2 m}}$. We are, however, unable to completely settle this issue at this point, though we can be sure that at least one of the containments is proper as Theorem 5.3 shows. Besides, it is currently computationally impossible to verify whether $\mathcal{G}$ in Example 5.2 is M-quasiconvex or not, whose answer should tell which proper containment in this chain is true or not. However, we believe that all these containing relationships are proper when $m \geq 3$, which leads to the following conjecture.

CONJECTURe 5.4. For $m \geq 3$ and $n \geq 2$, it holds that $\mathbb{S}_{2+}^{n^{2 m}} \subsetneq \mathbb{M}^{n^{2 m}} \subsetneq \mathbb{M}_{2}^{n^{2 m}}$.

6. Applications. Polynomial and tensor optimization is a rapidly expanding field. While its applications mushroomed in recent years, new solution methods remain relatively rare. In this section we aim to demonstrate a new scheme for polynomial optimization based on the notion of M-quasiconvexity. To begin, let us consider the following general constrained homogeneous polynomial optimization model

$$
\begin{array}{ll}
\max & f(\boldsymbol{x})=F(\underbrace{\boldsymbol{x}, \boldsymbol{x}, \ldots, \boldsymbol{x}}_{2 m}) \\
\text { s.t. } & \boldsymbol{x} \in S,
\end{array}
$$

where the constraint set $S \subseteq \mathbb{R}^{n}$ is compact. Essentially, the model is to find the largest eigenvalue of a symmetric tensor $\mathcal{F}$ over $S$. Based on the relationships among $\mathbb{S}_{2+}^{n^{2 m}}, \mathbb{M}^{n^{2 m}}$, and $\mathbb{M}_{2}^{n^{2 m}}$ discussed in previous sections, we have the following result. 
TheOREM 6.1. If $\mathcal{F} \in \mathbb{S}^{n^{2 m}}$ is M-quasiconvex, then for any $S \subseteq \mathbb{R}^{n}$

(L) $\begin{aligned} \max _{\boldsymbol{x} \in S} F(\underbrace{\boldsymbol{x}, \boldsymbol{x}, \ldots, \boldsymbol{x}}_{2 m}) & =\max _{\boldsymbol{x}_{1}, \boldsymbol{x}_{2}, \ldots, \boldsymbol{x}_{m} \in S} F\left(\boldsymbol{x}_{1}, \boldsymbol{x}_{1}, \boldsymbol{x}_{2}, \boldsymbol{x}_{2}, \ldots, \boldsymbol{x}_{m}, \boldsymbol{x}_{m}\right) \\ & =\max _{\boldsymbol{x}_{1}, \boldsymbol{x}_{2}, \ldots, \boldsymbol{x}_{2 m} \in S} F\left(\boldsymbol{x}_{1}, \boldsymbol{x}_{2}, \ldots, \boldsymbol{x}_{2 m}\right) . \quad(R)\end{aligned}$

Proof. Denote the optimal value of problems $(L),(M)$, and $(R)$ to be $v(L), v(M)$, and $v(R)$, respectively. As $(R)$ is a relaxation of $(M)$ and $(M)$ is a relaxation of $(L)$, we have that $v(L) \leq v(M) \leq v(R)$. It suffices to show $v(L) \geq v(R)$.

In fact, since $\mathcal{F} \in \mathbb{S}^{n^{2 m}}$ is M-quasiconvex, i.e.,

$$
F\left(\boldsymbol{x}_{1}, \boldsymbol{x}_{2}, \ldots, \boldsymbol{x}_{2 m}\right) \leq \max _{1 \leq i \leq 2 m}\left\{F(\underbrace{\boldsymbol{x}_{i}, \boldsymbol{x}_{i}, \ldots, \boldsymbol{x}_{i}}_{2 m})\right\} \quad \forall \boldsymbol{x}_{1}, \boldsymbol{x}_{2}, \ldots, \boldsymbol{x}_{2 m} \in S .
$$

Therefore $v(R) \leq v(L)$. This proves the theorem.

Theorem 6.1 establishes equivalence between homogeneous polynomial optimization and its multilinear form or multiquadratic form relaxation model over any constraint set, given that $\mathcal{F}$ is an M-quasiconvex tensor. Hence, as stated in section 4 of [8], Theorem 6.1 suggests an alternative way to deal with a homogeneous polynomial optimization model, say $(L)$. The general scheme, which consists of four main steps: lift, relax, block-optimize, and compare, named LRBC, is described as follows.

Algorithm 6.2 (LRBC).

1. Make sure the objective form is M-quasiconvex. Otherwise, lift it to an $M$-quasiconvex form if necessary, while keeping it equivalent to the original model $(L)$.

2. Relax the model $(L)$ to an equivalent multilinear form optimization model $(R)$, or a multiquadratic form optimization model $(M)$.

3. Apply any block optimization method (e.g., block coordinate descent (BCD), or maximum block improvement (MBI)) to solve the equivalent model $(R)$ or $(M)$.

4. Extract a stationary solution for the original model $(L)$ by choosing the best one among all the block components of the stationary solution for the equivalent model $(R)$ or $(M)$.

In the first step of LRBC, although it is in general hard to check whether a given symmetric tensor $\mathcal{F}$ is M-quasiconvex or not, there exist some sufficient conditions which are easily verifiable, e.g., matrix positive semidefinite described in Theorem 4.5. In case $\mathcal{F}$ is not M-quasiconvex (or at least not verified to be), as we will discuss shortly, in several applications it is possible to lift the tensor by transforming the objective into an M-quasiconvex one, while keeping the equivalence to the original model without affecting the optimal solution. In fact, it is often convenient to transform it into even stronger (sufficient) forms such as co-quadratic nonnegative or matrix positive semidefinite tensors; see Theorems 4.3 and 4.5. We will discuss the details of the lifting process in the application examples later in this section. Our numerical experiments in section 7 also suggest that the lifting technique is remarkably effective.

Once the M-quasiconvexity is satisfied, Theorem 6.1 guarantees the equivalence of multilinear relaxation $(R)$ or multiquadratic relaxation $(M)$ in the second step of LRBC. At a first glance, it might looks odd to consider $(M)$ or $(R)$ instead of $(L)$, as the latter contains a much smaller number of variables. The key observation however, is that $(M)$ or $(R)$ is convenient for block optimization: it is often very easy 
to optimize over one block of variables while fixing all others. In particular for $(L)$ the block optimization subproblem has a linear objective function. For block variable optimization, one choice for solving $(M)$ or $(R)$ is to implement the MBI method [8] or the BCD method [26] that usually finds a stationary solution (which in practice is often an optimal solution) quickly. The final step of LRBC is to construct a stationary solution (or an optimal solution) for the original problem. In this circumstance, suppose that $\left(\boldsymbol{x}_{1}, \boldsymbol{x}_{2}, \ldots, \boldsymbol{x}_{2 m}\right)$ is a stationary solution (or an optimal solution) for $(R)$, then we can directly find a stationary solution (or an optimal solution) $\boldsymbol{x}_{i^{*}}$ for $(L)$, where

$$
i^{*}=\arg \max _{1 \leq i \leq 2 m} F(\underbrace{\boldsymbol{x}_{i}, \boldsymbol{x}_{i}, \ldots, \boldsymbol{x}_{i}}_{2 m}),
$$

as the proof of Theorem 6.1 suggested. The best solution for $(L)$ is already among the solutions $\boldsymbol{x}_{1}, \boldsymbol{x}_{2}, \ldots, \boldsymbol{x}_{2 m}$. The procedure is much simpler as compared to Algorithm KKT proposed in section 4.2 of [8], thanks to the properties of M-quasiconvexity.

An immediate consequence of Theorem 6.1 is the following.

Corollary 6.3. If $\mathcal{F} \in \mathbb{S}^{n^{2 m}}$ is M-quasiconvex and integers $\lambda_{i} \geq 0(i=1,2$, $\ldots, s)$ with $\sum_{i=1}^{s} \lambda_{i}=2 m$, then for any $S \subseteq \mathbb{R}^{n}$

$$
\max _{\boldsymbol{x}_{1}, \boldsymbol{x}_{2}, \ldots, \boldsymbol{x}_{s} \in S} F(\underbrace{\boldsymbol{x}_{1}, \ldots, \boldsymbol{x}_{1}}_{\lambda_{1}}, \underbrace{\boldsymbol{x}_{2}, \ldots, \boldsymbol{x}_{2}}_{\lambda_{2}}, \ldots, \underbrace{\boldsymbol{x}_{s}, \ldots, \boldsymbol{x}_{s}}_{\lambda_{s}})=\max _{\boldsymbol{x} \in S} F(\underbrace{\boldsymbol{x}, \boldsymbol{x}, \ldots, \boldsymbol{x}}_{2 m}) .
$$

We remark that the constraint set $S$ is allowed to be arbitrary, which makes the scheme viable for a wide range of problems, including discrete optimization models. Let us present next some typical applications in polynomial and tensor optimization that are readily solved by the LRBC method based on M-quasiconvexity.

6.1. Tensor eigenvalue problem. The concept of eigenvalues/eigenvectors of tensors was proposed by Lim [24] and Qi [35] independently in 2005. In particular, finding the largest eigenvalue of a symmetric tensor $[35,36]$ is exactly the following spherical constrained homogeneous polynomial optimization problem:

$$
\begin{aligned}
& \text { (H) } \max \quad f(\boldsymbol{x})=F(\underbrace{\boldsymbol{x}, \boldsymbol{x}, \ldots, \boldsymbol{x}}_{d}) \\
& \text { s.t. }\|\boldsymbol{x}\|=1, \boldsymbol{x} \in \mathbb{R}^{n} \text {, }
\end{aligned}
$$

where $F$ is a multilinear form associated with a symmetric tensor $\mathcal{F} \in \mathbb{R}^{n^{d}}$. This problem has received much attention lately, not only for its fundamental properties but also for its wide applications, including numerical linear algebra, solid mechanic, signal processing, and quantum physics. It is also equivalent to the best rank-one approximation of a symmetric tensor; see e.g., [23].

A tensor relaxation method (relaxing to a multilinear form optimization model) for $(H)$ is the following:

$$
\begin{array}{lll}
\text { (T) } & \max & F\left(\boldsymbol{x}_{1}, \boldsymbol{x}_{2}, \ldots, \boldsymbol{x}_{d}\right) \\
& \text { s.t. } & \left\|\boldsymbol{x}_{i}\right\|=1, \boldsymbol{x}_{i} \in \mathbb{R}^{n}, i=1,2, \ldots, d .
\end{array}
$$

This relaxation is first proposed in [11] where the relationship between $(H)$ and $(T)$ as well as their approximate methods are discussed. Later, Chen et al. [8] and Zhang, Ling, and Qi [40] further explored this idea to solve $(H)$.

The study of the new classes of nonnegative tensors, however, gives us a much simpler way to deal with the model $(H)$ when the degree of the objective is even using 
the LRBC method. Rather than relaxing $(H)$ to $(T)$ directly, we first add a constant form $\alpha\left(\boldsymbol{x}^{\mathrm{T}} \boldsymbol{x}\right)^{m}=\alpha$ to the objective function, where $m=d / 2$ in $(H)$. By choosing $\alpha$ large enough we can ensure that the new objective function $f_{\alpha}(\boldsymbol{x}):=f(\boldsymbol{x})+$ $\alpha\left(\boldsymbol{x}^{\mathrm{T}} \boldsymbol{x}\right)^{m}$ is co-quadratic nonnegative, hence M-quasiconvex, which is guaranteed by the following result.

TheOREM 6.4. Denote $\mathcal{H} \in \mathbb{S}^{n^{2 m}}$ to be the symmetric tensor associated with the homogeneous polynomial function $\left(\boldsymbol{x}^{\mathrm{T}} \boldsymbol{x}\right)^{m}$. Then $\mathcal{H} \in \operatorname{int} \mathbb{S}_{2+}^{n^{2 m}}$ (the interior of $\mathbb{S}_{2+}^{n^{2 m}}$ ).

Proof. Denote $\mathbb{K}^{n^{2 m}}$ to be the convex hull of the set of symmetric rank-one tensors, i.e.,

$$
\mathbb{K}^{n^{2 m}}=\operatorname{conv}\{\mathcal{F} \in \mathbb{S}^{n^{2 m}} \mid \mathcal{F}=\underbrace{\boldsymbol{a} \circ \boldsymbol{a} \circ \cdots \circ \boldsymbol{a}}_{2 m}, \boldsymbol{a} \in \mathbb{R}^{n}\} .
$$

The proof is essentially based on the result that $\mathcal{H} \in \operatorname{int} \mathbb{K}^{n^{2 m}}$, which is stated as Theorem 8.15 in [37]. By noticing from Example 2.4 that any even order symmetric rank-one tensor is co-quadratic nonnegative, we have that any tensor in $\mathbb{K}^{n^{2 m}}$ is coquadratic nonnegative, and hence $\mathbb{K}^{n^{2 m}} \subseteq \mathbb{S}_{2+}^{n^{2 m}}$. This proves $\mathcal{H} \in \operatorname{int} \mathbb{S}_{2+}^{n^{2 m}}$.

In practice, for quartic tensor $\mathcal{F}$, letting $\alpha=3\|\mathcal{F}\|$ is sufficient to guarantee the co-quadratic nonnegativity of $\mathcal{F}+\alpha \mathcal{H}$. This is because

$$
H(\boldsymbol{x}, \boldsymbol{x}, \boldsymbol{y}, \boldsymbol{y})=\frac{1}{3}\left(\boldsymbol{x}^{\mathrm{T}} \boldsymbol{x}\right)\left(\boldsymbol{y}^{\mathrm{T}} \boldsymbol{y}\right)+\frac{2}{3}\left(\boldsymbol{x}^{\mathrm{T}} \boldsymbol{y}\right)^{2} \geq \frac{1}{3}\|\boldsymbol{x}\|^{2}\|\boldsymbol{y}\|^{2},
$$

implying that

$$
\begin{aligned}
(F+\alpha H)(\boldsymbol{x}, \boldsymbol{x}, \boldsymbol{y}, \boldsymbol{y}) & =F(\boldsymbol{x}, \boldsymbol{x}, \boldsymbol{y}, \boldsymbol{y})+\alpha H(\boldsymbol{x}, \boldsymbol{x}, \boldsymbol{y}, \boldsymbol{y}) \\
& \geq-\|\mathcal{F}\|\|\boldsymbol{x}\|^{2}\|\boldsymbol{y}\|^{2}+3\|\mathcal{F}\| \cdot \frac{1}{3}\|\boldsymbol{x}\|^{2}\|\boldsymbol{y}\|^{2} \\
& =0 .
\end{aligned}
$$

Another way to find $\alpha$ for a general degree tensor is to lift it to a matrix positive semidefinite tensor described in Theorem 4.5. This approach requires lifting with a parameter $\alpha>0$ such that $M(\mathcal{F}+\alpha \mathcal{H}) \succeq 0$. Such an $\alpha$ can be easily found by solving a semidefinite program.

After lifting the objective function of $(H)$ by $\alpha$, we can then equivalently reformulate the new homogeneous polynomial $f_{\alpha}(\boldsymbol{x})$ to its multilinear relaxation $(T)$, and apply a block optimization method to get a stationary (which is often optimal) solution. This equivalence is guaranteed by Theorem 6.1 since co-quadratic nonnegativity implies M-quasiconvexity. Finally, to return a solution to the original problem $(H)$, we only need to find the best $i^{*}$ with

$$
i^{*}=\arg \max _{1 \leq i \leq 2 m} F_{\alpha}(\underbrace{\boldsymbol{x}_{i}, \boldsymbol{x}_{i}, \ldots, \boldsymbol{x}_{i}}_{2 m}),
$$

thanks to the M-quasiconvexity of $\mathcal{F}_{\alpha}$ guaranteed by its co-quadratic nonnegativity (Theorem 4.3).

Indeed according to LRBC, we can also equivalently transfer $(H)$ to a multiquadratic form optimization after its objective function is lifted to $f_{\alpha}(\boldsymbol{x})$,

$$
\begin{array}{lll}
\text { (Q) } & \max & F_{\alpha}\left(\boldsymbol{x}_{1}, \boldsymbol{x}_{1}, \boldsymbol{x}_{2}, \boldsymbol{x}_{2}, \ldots, \boldsymbol{x}_{m}, \boldsymbol{x}_{m}\right) \\
& \text { s.t. } & \left\|\boldsymbol{x}_{i}\right\|=1, \boldsymbol{x}_{i} \in \mathbb{R}^{n}, i=1,2, \ldots, m
\end{array}
$$


This relaxation can also be solved by resorting to block optimization (fixing $m-1$ blocks and optimizing one block at each time), e.g., the MBI or the BCD method as a subroutine, which in this case reduces to a matrix eigenvalue problem. Moreover, after obtaining a solution for $(Q)$, we are able to return a solution for $(H)$ by choosing

$$
i^{*}=\arg \max _{1 \leq i \leq m} F_{\alpha}(\underbrace{\boldsymbol{x}_{i}, \boldsymbol{x}_{i}, \ldots, \boldsymbol{x}_{i}}_{2 m})
$$

because of the co-quadratic M-quasiconvexity of $\mathcal{F}_{\alpha}$, which is guaranteed by its co-quadratic nonnegativity (Theorem 4.2).

6.2. Biquadratic model in circuit design. The quadratic assignment problem (QAP) is known as one of the most challenging problems in combinatorial optimization. Recently, there have been attempts to solve the QAP via semidefinite programming relaxations as a lower bounding procedure; see, e.g., [9, 19, 33]. The biquadratic assignment problem (BiQAP) is a generalization of the QAP, which is to minimize a quartic polynomial of an assignment matrix:

$$
\begin{array}{ll}
\min & \sum_{1 \leq i, j, k, \ell, s, t, u, v \leq n} \mathcal{A}_{i j k \ell} \mathcal{B}_{s t u v} X_{i s} X_{j t} X_{k u} X_{\ell v} \\
\text { s.t. } & \sum_{j=1}^{n} X_{i j}=1, i=1,2, \ldots, n \\
& \sum_{i=1}^{n} X_{i j}=1, j=1,2, \ldots, n \\
& X_{i j} \in\{0,1\}, i, j=1,2, \ldots, n \\
& X \in \mathbb{R}^{n \times n}
\end{array}
$$

where $\mathcal{A}, \mathcal{B} \in \mathbb{R}^{n \times n \times n \times n}$. Motivated by a practical application in the very large scale integrated synthesis problem, BiQAP was first introduced and studied by Burkard, Cela, and Klinz, [7]. Several heuristics for the BiQAP were subsequently developed by Burkard and Cela [6] and Mavridou et al. [29].

The objective function of the BiQAP is a fourth degree polynomial function of the variables $X_{i j}$ 's, where $X$ is taken as an $n^{2}$-dimensional vector. In particular, by denoting $\boldsymbol{x}:=\operatorname{vec}(X) \in \mathbb{R}^{n^{2}}$, we can find a quartic form $F$ associated with a symmetric quartic tensor $\mathcal{F}:=\operatorname{sym}(-\mathcal{A} \otimes \mathcal{B}) \in \mathbb{S}^{\left(n^{2}\right)^{4}}$, where the notation "sym" symmetrizes a tensor, such that

$$
F(\boldsymbol{x}, \boldsymbol{x}, \boldsymbol{x}, \boldsymbol{x}):=\sum_{1 \leq i, j, k, \ell, s, t, u, v \leq n}-\mathcal{A}_{i j k \ell} \mathcal{B}_{s t u v} X_{i s} X_{j t} X_{k u} X_{\ell v} .
$$

For the constraints, one finds vectors $\boldsymbol{a}_{1}, \boldsymbol{a}_{2}, \ldots, \boldsymbol{a}_{n}, \boldsymbol{b}_{1}, \boldsymbol{b}_{2}, \ldots, \boldsymbol{b}_{n} \in \mathbb{R}^{n^{2}}$ in such a way that

$$
\begin{gathered}
\boldsymbol{a}_{i}^{\mathrm{T}} \boldsymbol{x}=\sum_{j=1}^{n} X_{i j}=1, i=1,2, \ldots, n, \\
\boldsymbol{b}_{j}^{\mathrm{T}} \boldsymbol{x}=\sum_{i=1}^{n} X_{i j}=1, j=1,2, \ldots, n .
\end{gathered}
$$

We have the equivalent formulation of the BiQAP,

$$
\begin{array}{ll}
\max & F(\boldsymbol{x}, \boldsymbol{x}, \boldsymbol{x}, \boldsymbol{x}) \\
\mathrm{s.t.} & \boldsymbol{a}_{i}^{\mathrm{T}} \boldsymbol{x}=1, i=1,2, \ldots, n, \\
& \boldsymbol{b}_{j}^{\mathrm{T}} \boldsymbol{x}=1, j=1,2, \ldots, n, \\
& \boldsymbol{x} \in\{0,1\}^{n^{2}} .
\end{array}
$$


An important observation is that $\boldsymbol{x}^{\mathrm{T}} \boldsymbol{x}=n$ as $X$ is an assignment matrix. Thus the objective function of the above model can be lifted up to a co-quadratic nonnegative form by adding a constant term $\alpha\left(\boldsymbol{x}^{\mathrm{T}} \boldsymbol{x}\right)^{2}$ according to Theorem 6.4. Using the approaches mentioned in section 6.1, we can find $\alpha$ to guarantee the co-quadratic nonnegativity of $F_{\alpha}(\boldsymbol{x}, \boldsymbol{x}, \boldsymbol{x}, \boldsymbol{x}):=F(\boldsymbol{x}, \boldsymbol{x}, \boldsymbol{x}, \boldsymbol{x})+\alpha\left(\boldsymbol{x}^{\mathrm{T}} \boldsymbol{x}\right)^{2}$, which implies that it is M-quasiconvex and establishes the first step of the LRBC method. By Theorem 6.1, the BiQAP can then be reformulated in the multilinear form model

$$
\begin{array}{ll}
\max & F_{\alpha}(\boldsymbol{x}, \boldsymbol{y}, \boldsymbol{z}, \boldsymbol{w}) \\
\mathrm{s.t.} & \boldsymbol{a}_{i}^{\mathrm{T}} \boldsymbol{x}=\boldsymbol{a}_{i}^{\mathrm{T}} \boldsymbol{y}=\boldsymbol{a}_{i}^{\mathrm{T}} \boldsymbol{z}=\boldsymbol{a}_{i}^{\mathrm{T}} \boldsymbol{w}=1, i=1,2, \ldots, n, \\
& \boldsymbol{b}_{j}^{\mathrm{T}} \boldsymbol{x}=\boldsymbol{b}_{j}^{\mathrm{T}} \boldsymbol{y}=\boldsymbol{b}_{j}^{\mathrm{T}} \boldsymbol{z}=\boldsymbol{b}_{j}^{\mathrm{T}} \boldsymbol{w}=1, j=1,2, \ldots, n, \\
& \boldsymbol{x}, \boldsymbol{y}, \boldsymbol{z}, \boldsymbol{w} \in\{0,1\}^{n^{2}} .
\end{array}
$$

Note that the subproblem of the above optimization model (fixing 3 blocks and optimizing one block) is a linear assignment, which is easily solvable. Therefore a block optimization method such as BCD or MBI can be used to solve this model and get a stationary solution $\left(\boldsymbol{x}^{*}, \boldsymbol{y}^{*}, \boldsymbol{z}^{*}, \boldsymbol{w}^{*}\right)$. Finally,

$$
\arg \max \left\{F_{\alpha}\left(\boldsymbol{x}^{*}, \boldsymbol{x}^{*}, \boldsymbol{x}^{*}, \boldsymbol{x}^{*}\right), F_{\alpha}\left(\boldsymbol{y}^{*}, \boldsymbol{y}^{*}, \boldsymbol{y}^{*}, \boldsymbol{y}^{*}\right), F_{\alpha}\left(\boldsymbol{z}^{*}, \boldsymbol{z}^{*}, \boldsymbol{z}^{*}, \boldsymbol{z}^{*}\right), F_{\alpha}\left(\boldsymbol{w}^{*}, \boldsymbol{w}^{*}, \boldsymbol{w}^{*}, \boldsymbol{w}^{*}\right)\right\}
$$

is a local stationary solution to the original BiQAP, as guaranteed by the M-quasiconvexity of $\mathcal{F}_{\alpha}$.

6.3. Portfolio selection with higher moments. In modern portfolio management, the celebrated mean-variance model was originally introduced by Markowitz [28] back in 1952, where the portfolio selection problem is modeled by minimizing the variance of the return of the portfolio for a given level of its expected return, as follows:

$$
\begin{array}{ll}
\min & \boldsymbol{x}^{\mathrm{T}} \Sigma \boldsymbol{x} \\
\mathrm{s.t.} & \boldsymbol{\mu}^{\mathrm{T}} \boldsymbol{x}=\mu_{0}, \\
& \boldsymbol{e}^{\mathrm{T}} \boldsymbol{x}=1, \\
& \boldsymbol{x} \in \mathbb{R}^{n},
\end{array}
$$

where $\boldsymbol{\mu}$ and $\Sigma$ are the mean vector and covariance matrix of $n$ given assets, respectively, and $\boldsymbol{e}$ is the all-ones vector. Much of the mean variance theory has been focusing on the first two moments of the return of the portfolio. Recently, the framework of mean variance has been extended to include the skewness and kurtosis information; see, e.g., Jondeau and Rockinger [17], Kleniati, Parpas, and Rustem [18], Maringer and Parpas [27] and the references therein.

Let us now consider a similar model proposed in [30] which minimizes the kurtosis under the constraints of the mean and variance of the portfolio, as follows:

$$
\begin{array}{cl}
(K) \min & F(\boldsymbol{x}, \boldsymbol{x}, \boldsymbol{x}, \boldsymbol{x}) \\
\mathrm{s.t.} & \boldsymbol{x}^{\mathrm{T}} \Sigma \boldsymbol{x}=\sigma^{2}, \\
& \boldsymbol{\mu}^{\mathrm{T}} \boldsymbol{x} \geq \mu_{0}, \\
& \boldsymbol{e}^{\mathrm{T}} \boldsymbol{x}=1, \\
& \boldsymbol{x} \in S,
\end{array}
$$

where $S$ can be $\mathbb{R}^{n}$ or $\mathbb{R}_{+}^{n}$ depending on if short selling is allowed or not. Toward the solution method, we may let $\boldsymbol{y}=\frac{1}{\sigma} \sum^{\frac{1}{2}} \boldsymbol{x}$ since the covariance matrix $\Sigma$ is positive 
semidefinite. Hence $\boldsymbol{x}=\sigma \Sigma^{-\frac{1}{2}} \boldsymbol{y}$ (use the Moore-Penrose inverse if $\Sigma$ is not positive definite). We can reformulate $(K)$ to the following equivalent model:

$$
\begin{aligned}
\left(K^{\prime}\right) \quad \max & F^{\prime}(\boldsymbol{y}, \boldsymbol{y}, \boldsymbol{y}, \boldsymbol{y})=-\sigma^{4} F\left(\Sigma^{-\frac{1}{2}} \boldsymbol{y}, \Sigma^{-\frac{1}{2}} \boldsymbol{y}, \Sigma^{-\frac{1}{2}} \boldsymbol{y}, \Sigma^{-\frac{1}{2}} \boldsymbol{y}\right) \\
\text { s.t. } \quad & \boldsymbol{y}^{\mathrm{T}} \boldsymbol{y}=1, \\
& \sigma \boldsymbol{\mu}^{\mathrm{T}} \Sigma^{-\frac{1}{2}} \boldsymbol{y} \geq \mu_{0} \\
& \sigma \boldsymbol{e}^{\mathrm{T}} \Sigma^{-\frac{1}{2}} \boldsymbol{y}=1 \\
& \boldsymbol{y} \in S^{\prime}
\end{aligned}
$$

where $S^{\prime}$ is either $\mathbb{R}^{n}$ or some linear inequalities $\Sigma^{-\frac{1}{2}} \boldsymbol{y} \in \mathbb{R}_{+}^{n}$. Noticing that $\boldsymbol{y}^{\mathrm{T}} \boldsymbol{y}=1$ and using a similar lift method introduced in the previous subsections, we can change the objective function of $\left(K^{\prime}\right)$ to a co-quadratic nonnegative form $F^{\prime}(\boldsymbol{y}, \boldsymbol{y}, \boldsymbol{y}, \boldsymbol{y})+$ $\alpha\left(\boldsymbol{y}^{\mathrm{T}} \boldsymbol{y}\right)^{2}$. Then we can reformulate it to a multilinear form optimization model as Theorem 6.1 stipulated. The solution of $\left(K^{\prime}\right)$ can be obtained by applying the MBI or the BCD method to solve the multilinear model and comparing the objective $F^{\prime}$ among the four blocks of variables in the solution of the multilinear model, as the M-quasiconvexity of the form $F^{\prime}(\boldsymbol{y}, \boldsymbol{y}, \boldsymbol{y}, \boldsymbol{y})+\alpha\left(\boldsymbol{y}^{\mathrm{T}} \boldsymbol{y}\right)^{2}$ enables us to relax the coupled variables to an easier looking but equivalent block optimization model.

7. Numerical experiments. In this section, we present some numerical results testing the practical performance of the new technique. All the numerical experiments are conducted on an Intel Xeon CPU $3.40 \mathrm{GHz}$ computer with 8GB RAM. The supporting software is MATLAB 7.12.0 (R2011a) as a platform. We use MATLAB Tensor Toolbox v2.5 [2] whenever tensor operations are called.

We experiment with two types of polynomial optimization models: (1) portfolio selection with higher moments as discussed in section 6.3 where historical data are used; (2) homogeneous quartic polynomial optimization over quadratic constraints where simulated data are used. In our implementations, the objective homogenous polynomial is either M-quasiconvex or can be equivalently transformed to an Mquasiconvex one, which paves the way to apply the LRBC scheme. In the block optimization step of the LRBC procedure, we apply the MBI method to the portfolio selection example, and the BCD method to the quadratically constrained quartic polynomial optimization model. The subproblems in the block optimization step (optimizing one block while fixing all other blocks) for both models can be equivalently formulated as convex optimization models, where cvx v1.2 (Grant and Boyd [10]) is called to solve these subroutines. To measure the quality of the solutions obtained by our approaches, we compare them to the true global optimal solutions obtained by a general-purpose method for polynomial optimization, in this particular case GloptiPoly 3 [13] is called and its relaxation order is set by default.

7.1. Portfolio selection with higher moments. Let us revisit section 6.3 and consider the model $(K)$ for numerical experiments. Forty stocks listed in the New York Stock Exchange are considered and their adjusted close prices ranging from March 1, 2014 to March 1, 2015 are drawn from Yahoo Finance. For $n=10,20,30,40$ in $(K)$, its first, second, and fourth moments are computed, corresponding to the mean vector $\boldsymbol{\mu} \in \mathbb{R}^{n}$, the covariance matrix $\Sigma \in \mathbb{S}^{n^{2}}$, and the kurtosis tensor $\mathcal{F} \in \mathbb{S}^{n^{4}}$, respectively. The set $S$ is defined to be $\mathbb{R}^{n}$, i.e., short selling is allowed. 
To apply our method based on the theory of M-quasiconvexity, we reformulate $(K)$ as $\left(K^{\prime}\right)$ discussed in section 6.3 :

$$
\begin{aligned}
\left(K^{\prime}\right) \quad \max & F^{\prime}(\boldsymbol{y}, \boldsymbol{y}, \boldsymbol{y}, \boldsymbol{y})=-\sigma^{4} F\left(\Sigma^{-\frac{1}{2}} \boldsymbol{y}, \Sigma^{-\frac{1}{2}} \boldsymbol{y}, \Sigma^{-\frac{1}{2}} \boldsymbol{y}, \Sigma^{-\frac{1}{2}} \boldsymbol{y}\right) \\
\text { s.t. } & \boldsymbol{y}^{\mathrm{T}} \boldsymbol{y}=1, \\
& \sigma \boldsymbol{\mu}^{\mathrm{T}} \Sigma^{-\frac{1}{2}} \boldsymbol{y} \geq \mu_{0} \\
& \sigma \boldsymbol{e}^{\mathrm{T}} \Sigma^{-\frac{1}{2}} \boldsymbol{y}=1 \\
& \boldsymbol{y} \in \mathbb{R}^{n} .
\end{aligned}
$$

In the first step of LRBC, we add a constant $3\left\|\mathcal{F}^{\prime}\right\|$ to $F^{\prime}(\boldsymbol{y}, \boldsymbol{y}, \boldsymbol{y}, \boldsymbol{y})$ and the objective function is lifted to

$$
G(\boldsymbol{y}, \boldsymbol{y}, \boldsymbol{y}, \boldsymbol{y}):=F^{\prime}(\boldsymbol{y}, \boldsymbol{y}, \boldsymbol{y}, \boldsymbol{y})+3\left\|\mathcal{F}^{\prime}\right\|\left(\boldsymbol{y}^{\mathrm{T}} \boldsymbol{y}\right)^{2},
$$

which is M-quasiconvex as discussed in section 6.1. We then equivalently formulate the lifted problem to the multilinear model $\left(K_{1}\right)$, as guaranteed by Theorem 6.1:

$$
\begin{array}{lll}
\left(K_{1}\right) & \max & G\left(\boldsymbol{x}_{1}, \boldsymbol{x}_{2}, \boldsymbol{x}_{3}, \boldsymbol{x}_{4}\right) \\
& \text { s.t. } & \boldsymbol{x}_{1}, \boldsymbol{x}_{2}, \boldsymbol{x}_{3}, \boldsymbol{x}_{4} \in S^{\prime},
\end{array}
$$

where $S^{\prime}:=\left\{\boldsymbol{y} \in \mathbb{R}^{n}: \boldsymbol{y}^{\mathrm{T}} \boldsymbol{y}=1, \sigma \boldsymbol{\mu}^{\mathrm{T}} \Sigma^{-\frac{1}{2}} \boldsymbol{y} \geq \mu_{0}, \sigma \boldsymbol{e}^{\mathrm{T}} \Sigma^{-\frac{1}{2}} \boldsymbol{y}=1\right\}$. The MBI method is applied to solve the block optimization model $\left(K_{1}\right)$, starting from any feasible solution $\left(\boldsymbol{x}_{1}, \boldsymbol{x}_{2}, \boldsymbol{x}_{3}, \boldsymbol{x}_{4}\right)$ and optimize one block $\boldsymbol{x}_{k}$ while fixing the other three blocks $\boldsymbol{x}_{i}$ 's. Here, the subproblem is maximizing a linear form over $S^{\prime}$, which is equivalent to the maximization over $\left\{\boldsymbol{y} \in \mathbb{R}^{n}: \boldsymbol{y}^{\mathrm{T}} \boldsymbol{y} \leq 1, \sigma \boldsymbol{\mu}^{\mathrm{T}} \Sigma^{-\frac{1}{2}} \boldsymbol{y} \geq \mu_{0}, \sigma \boldsymbol{e}^{\mathrm{T}} \Sigma^{-\frac{1}{2}}\right.$ $\boldsymbol{y}=1$, i.e., replacing the spherical constraint to the ball constraint. This reformulated subproblem is convex, and can be easily solved, e.g., using cvx.

For the purpose of showing the effect of lifting the objective function in the first step of LRBC, we also apply the MBI method to solve the multilinear form relaxation model of $\left(K^{\prime}\right)$ directly without lifting (7.1):

$$
\begin{array}{lll}
\left(K_{2}\right) & \max & F^{\prime}\left(\boldsymbol{x}_{1}, \boldsymbol{x}_{2}, \boldsymbol{x}_{3}, \boldsymbol{x}_{4}\right) \\
& \text { s.t. } & \boldsymbol{x}_{1}, \boldsymbol{x}_{2}, \boldsymbol{x}_{3}, \boldsymbol{x}_{4} \in S^{\prime} .
\end{array}
$$

When applying the MBI method to solve $\left(K_{1}\right)$ and $\left(K_{2}\right)$, we begin with the same initial solutions, which are randomly generated in $S^{\prime}$. The termination precision is set to be $10^{-4}$. Unlike the equivalence between $\left(K^{\prime}\right)$ and $\left(K_{1}\right)$ from Theorem $6.1,\left(K_{2}\right)$ and $\left(K^{\prime}\right)$ may not be equivalent. Denote $\left(\boldsymbol{x}_{1}^{*}, \boldsymbol{x}_{2}^{*}, \boldsymbol{x}_{3}^{*}, \boldsymbol{x}_{4}^{*}\right)$ to be a stationary solution found by the MBI method for $\left(K_{1}\right)$ or $\left(K_{2}\right)$. Our numerical results show that these four blocks are always identical for $\left(K_{1}\right)$, i.e., $\boldsymbol{x}_{1}^{*}=\boldsymbol{x}_{2}^{*}=\boldsymbol{x}_{3}^{*}=\boldsymbol{x}_{4}^{*}$, but they are always not equal to each other for $\left(K_{2}\right)$. In the latter case, the solution for $\left(K^{\prime}\right)$ is chosen as the best one among those four vectors, namely, $\boldsymbol{y}^{*}=\arg \max _{1 \leq i \leq 4}\left\{F^{\prime}\left(\boldsymbol{x}_{i}^{*}, \boldsymbol{x}_{i}^{*}, \boldsymbol{x}_{i}^{*}, \boldsymbol{x}_{i}^{*}\right)\right\}$, as suggested in the last step of LRBC.

Numerical results are summarized in Table 1 . To validate the solution qualities of both the lifted and unlifted approaches, GloptiPoly 3 is chosen as the global method to solve $(K)$ directly. For all the cases, we report the (local) optimal value or its lower bound (if GloptiPoly 3 fails to provide an optimal certificate) of the original problem $(K)$. Values in boldface indicate that the global method guarantees the output objective value to be optimal. For the block optimization step of LRBC in each instance, we run the MBI method 10 times, starting from 10 different initial points. The column Value denotes the range of these objective values, with the left end being 
TABLE 1

Numerical results for portfolio optimization.

\begin{tabular}{|c|c|c|c|c|c|c|}
\hline \multirow[t]{2}{*}{$n$} & \multirow[t]{2}{*}{$\left(\mu_{0}, \sigma\right)$} & \multirow{2}{*}{$\frac{\text { Global method }}{\text { Value }}$} & \multicolumn{3}{|c|}{ LRBC } & \multirow{2}{*}{$\frac{\text { Unlifted }}{\text { Value (mean) }}$} \\
\hline & & & Value (range) & Value (mean) & CPU (mean) & \\
\hline \multirow[t]{2}{*}{$\overline{10}$} & $(0.05,2)$ & 43.80 & {$[43.82,47.08]$} & 45.41 & $1.04 \mathrm{e}+03$ & 95.75 \\
\hline & $(0.10,2)$ & 44.50 & {$[44.51,49.52]$} & 46.48 & $0.76 \mathrm{e}+03$ & 95.80 \\
\hline \multirow[t]{2}{*}{20} & $(0.05,2)$ & 35.27 & {$[35.36,37.23]$} & 36.23 & $2.00 \mathrm{e}+03$ & 147.78 \\
\hline & $(0.10,2)$ & 35.55 & {$[35.73,37.83]$} & 36.86 & $1.83 \mathrm{e}+03$ & 156.80 \\
\hline \multirow[t]{2}{*}{30} & $(0.05,2)$ & - & {$[29.26,34.90]$} & 30.63 & $3.32 \mathrm{e}+03$ & 116.61 \\
\hline & $(0.10,2)$ & - & {$[29.25,31.11]$} & 29.83 & $4.86 \mathrm{e}+03$ & 160.36 \\
\hline \multirow[t]{2}{*}{40} & $(0.05,2)$ & - & {$[26.87,29.64]$} & 27.84 & $4.47 \mathrm{e}+03$ & 225.42 \\
\hline & $(0.10,2)$ & - & {$[26.23,29.78]$} & 27.82 & $4.92 \mathrm{e}+03$ & 166.10 \\
\hline
\end{tabular}

the best recorded solution and the right end being the worst recorded solution among these 10. We also report the average computational time in CPU among these 10 trials. The column Unlifted denotes the results for solving the unlifted model $\left(K_{2}\right)$, i.e., without the lifting step in LRBC.

One observes that the LRBC approach almost always finds global optima guaranteed by GloptiPoly 3 when $n=10$ by choosing the best one among the 10 trials in the block optimization step. For $n=20$, the lower bound provided by GloptiPoly 3 and local optimal value found by LRBC are very close. This implies that GloptiPoly 3 and our new method, in collaboration, produce solutions with an optimality certificate. In the case when high precision is needed, one usually runs more trials in the block optimization step and chooses the best one. This will certainly improve the solution quality of LRBC. Unfortunately, there is no global method that can handle problems with size $n \geq 30$ to validate the solution quality of LRBC, but we believe it is still very good from the results for $n \leq 20$. For the computational cost, LRBC is almost linear in terms of the dimension $n$. These observations imply that LRBC can solve large scale problems in reasonable computational time with high solution quality. As observed before, the values of the unlifted model do not lead to optimal solutions at all. This means that equivalently turning the model into the M-quasiconvexity one is really the key for the lifting and relaxation approach to work well.

7.2. Homogeneous polynomial optimization over quadratic constraints. To extensively test our method, we now use randomly generated datasets. The model considered here is to maximize a convex quartic form over the intersection of cocentered ellipsoids:

$$
\begin{array}{ll}
\text { (E) } \max & F(\boldsymbol{x}, \boldsymbol{x}, \boldsymbol{x}, \boldsymbol{x}) \\
\text { s.t. } & \boldsymbol{x}^{\mathrm{T}} Q_{j} \boldsymbol{x} \leq 1, j=1,2, \ldots, m, \\
& \boldsymbol{x} \in \mathbb{R}^{n}
\end{array}
$$

where the tensor $\mathcal{F}$ is symmetric and M-quasiconvex, the matrix $Q_{j}$ is positive semidefinite for $j=1,2, \ldots, m$, and $\sum_{j=1}^{m} Q_{j}$ is positive definite.

By the M-quasiconvexity (or convexity) of $\mathcal{F}$, LRBC can be easily applied to solve $(E)$, whose equivalent multilinear form relaxation is

$$
\begin{array}{lll}
\left(E_{1}\right) & \max & F\left(\boldsymbol{x}_{1}, \boldsymbol{x}_{2}, \boldsymbol{x}_{3}, \boldsymbol{x}_{4}\right) \\
& \text { s.t. } & \boldsymbol{x}_{i}^{\mathrm{T}} Q_{j} \boldsymbol{x}_{i} \leq 1, i=1,2,3,4, j=1,2, \ldots, m, \\
& \boldsymbol{x}_{i} \in \mathbb{R}^{n}, i=1,2,3,4 .
\end{array}
$$


TABLE 2

Numerical results for solving $(E)$ when $m=10$.

\begin{tabular}{lcccccccc}
\hline$n$ & \# samples & \multicolumn{2}{c}{ Global method } & & \multicolumn{3}{c}{ LRBC } & Gap (mean) \\
\cline { 3 - 4 } & & \# opt & CPU (mean) & & \# opt & \# cert & CPU (mean) & \\
\hline 5 & 100 & 90 & 0.3 & & 89 & 3 & 61.2 & 0.001333 \\
10 & 100 & 98 & 4.3 & & 95 & 2 & 159.2 & 0.001862 \\
15 & 100 & 95 & 190.6 & & 94 & 4 & 246.8 & 0.000128 \\
\hline
\end{tabular}

We use the cyclic (alternatively updating) BCD method to solve $\left(E_{1}\right)$ in the block optimization step of LRBC. The relevant subproblem in question is in the form of

$$
\begin{array}{ll}
\max & \boldsymbol{u}^{\mathrm{T}} \boldsymbol{x}_{i} \\
\mathrm{s.t.} & \boldsymbol{x}_{i}^{\mathrm{T}} Q_{j} \boldsymbol{x}_{i} \leq 1, j=1,2, \ldots, m, \\
& \boldsymbol{x}_{i} \in \mathbb{R}^{n},
\end{array}
$$

and it can be formulated as a second order cone program; we use cvx to solve these subproblems in our implementation.

The data for $(E)$ are generated in the following manner. First, a fourth order tensor with its $n^{4}$ entries following i.i.d. standard normal distributions are generated and then symmetrized by averaging the corresponding entries, denoted by $\mathcal{F}^{\prime}$. Next, we let $\mathcal{F}=\mathcal{F}^{\prime}+3\left\|\mathcal{F}^{\prime}\right\| \mathcal{H}$, where $\mathcal{H} \in \mathbb{S}^{n^{4}}$ is the symmetric tensor associated with the homogeneous polynomial $\left(\boldsymbol{x}^{\mathrm{T}} \boldsymbol{x}\right)^{2}$ as discussed in Theorem 6.4. In this setting, $\mathcal{F}$ is convex and hence M-quasiconvex. For the matrix $Q_{j}(j=1,2, \ldots, m)$, we first generate $Q_{j}^{\prime}$ whose entries follow i.i.d. standard normal distribution, and then let $Q_{j}=\left(Q_{j}^{\prime}\right)^{\mathrm{T}} Q_{j}^{\prime}$ for $j=1,2, \ldots, m$.

In the block optimization step of LRBC, we run the BCD method 10 times from 10 diffident randomly generated starting solutions and pick the best one when the algorithm stops. The termination precision is set to be $10^{-6}$. Table 2 presents the detailed performance of the solution quality for the global method (GloptiPoly 3) and LRBC when $m=10$ and $n$ is small for which GloptiPoly 3 is applicable. For GloptiPoly 3, if it works then it either outputs the optimal value or an upper bound. A list of abbreviations to understand Table 2 is as follows:

- \# samples: The total number of randomly generated instances of $(E)$.

- \# opt: The number of instances that GloptiPoly 3 guarantees the optimality or the solution found by LRBC attains the optimal value assured by GloptiPoly 3 .

- \# cert: The number of instances that the solutions by LRBC attain the upper bounds produced by GloptiPoly 3, hence certifying that the upper bounds of GloptiPoly 3 are actually optimal.

- Gap (mean): The average gap between the solution by LRBC and the optimal value or its upper bound by GloptiPoly 3 .

In running the tests, we observe that the BCD method for solving $\left(E_{1}\right)$ always outputs its stationary solution $\left(\boldsymbol{x}_{1}, \boldsymbol{x}_{2}, \boldsymbol{x}_{3}, \boldsymbol{x}_{4}\right)$ satisfying $\boldsymbol{x}_{1}= \pm \boldsymbol{x}_{2}= \pm \boldsymbol{x}_{3}= \pm \boldsymbol{x}_{4}$, meaning that $\boldsymbol{x}_{1}$ is a local maxima of the original problem $(E)$. Remark that we can always swap the direction from $\boldsymbol{x}_{1}$ to $-\boldsymbol{x}_{1}$ without affecting its objective value. This again shows the importance of the M-quasiconvexity, which is the usual convexity here for quartic forms. From Table 2, we see that the quality of LRBC is evident from the optimality certificates of GloptiPoly 3. In addition, in most of the instances that GloptiPoly 3 fails to provide an optimality certificate, the optimality 
TABLE 3

Average CPU seconds for solving $(E)$ when $m=15$.

\begin{tabular}{crrrrrcccc}
\hline$n$ & 5 & 10 & 15 & 20 & 30 & 40 & 50 & 70 & 100 \\
\hline LRBC & 44 & 199 & 312 & 542 & 780 & $1.18 \mathrm{e}+03$ & $1.43 \mathrm{e}+03$ & $2.25 \mathrm{e}+03$ & $5.63 \mathrm{e}+03$ \\
\hline
\end{tabular}

is actually certified by the solution of LRBC as its objective value equals the upper bound provided by GloptiPoly 3 (\# Cert).

Finally, let us report the computational time of the LRBC method. Although it is slower than GloptiPoly 3 for low dimension problems as shown in Table 2, it is actually insensitive to the problem dimensions. In Table 3 , we fix $m=15$ for various $n$, and present the performance of the LRBC method in terms of CPU time. Each entry is the average computational time of 30 randomly generated instances. Although the computational time is highly dependent on the number of trials for performing the block optimization step in LRBC (in Table 3 it is 10 trials for each instance), it is actually observed to be linear in terms of the problem dimension.

To summarize, our numerical experiments show that for certain classes of polynomial optimization models where the M-quasiconvexity can be guaranteed (equivalently transformed into) then the LRBC approach is found to be able to generate high quality solutions efficiently, even for large scale problems. For small size problems, the solution's qualities are comparable to that of globally optimal ones. The computational cost is observed to be linear in terms of the dimension of the model.

8. Concluding remarks. In this paper we study three new classes of nonnegative tensors: co-quadratic nonnegativity, M-quasiconvexity, and co-quadratic M-quasiconvexity, respectively. We also discuss their relationships. We demonstrate that the M-quasiconvexity plays an important role in linking homogeneous polynomial optimization problems to their tensor relaxation counterparts. As a result, this makes it possible to solve polynomial optimization models by means of block coordinate search methods. Finally, we remark that it is possible to extend the notion of co-quadratic nonnegativity and M-quasiconvexity to more general settings. As an example, consider the following extension.

DEFINITION 8.1. Suppose that $F$ is a multilinear form associated with a symmetric tensor $\mathcal{F} \in \mathbb{S}^{n^{t m}}$ of order tm, where $t$ and $m$ are two positive integers. The tensor $\mathcal{F}$ (or the form $F$ ) is called co-nonnegative of order $t$ over $S \subseteq \mathbb{R}^{n}$ if

$$
F(\underbrace{\boldsymbol{x}_{1}, \ldots, \boldsymbol{x}_{1}}_{t}, \underbrace{\boldsymbol{x}_{2}, \ldots, \boldsymbol{x}_{2}}_{t}, \ldots, \underbrace{\boldsymbol{x}_{m}, \ldots, \boldsymbol{x}_{m}}_{t}) \geq 0 \quad \forall \boldsymbol{x}_{1}, \boldsymbol{x}_{2}, \ldots, \boldsymbol{x}_{m} \in S .
$$

DEFINITION 8.2. Suppose that $F$ is a multilinear form associated with a symmetric tensor $\mathcal{F} \in \mathbb{S}^{n^{t m}}$ of order tm, where $t$ and $m$ are two positive integers. The tensor $\mathcal{F}$ is called $M$-quasiconvex of order $t$ over $S \subseteq \mathbb{R}^{n}$ if

$$
\begin{aligned}
& F(\underbrace{\boldsymbol{x}_{1}, \ldots, \boldsymbol{x}_{1}}_{t}, \underbrace{\boldsymbol{x}_{2}, \ldots, \boldsymbol{x}_{2}}_{t}, \ldots, \underbrace{\boldsymbol{x}_{m}, \ldots, \boldsymbol{x}_{m}}_{t}) \\
& \leq \max _{1 \leq i \leq m}\left\{F(\underbrace{\boldsymbol{x}_{i}, \boldsymbol{x}_{i}, \ldots, \boldsymbol{x}_{i}}_{t m})\right\} \quad \forall \boldsymbol{x}_{1}, \boldsymbol{x}_{2}, \ldots, \boldsymbol{x}_{m} \in S .
\end{aligned}
$$

Therefore, co-quadratic nonnegativity is co-nonnegativity of order 2 over $\mathbb{R}^{n}$, and M-quasiconvexity and co-quadratic M-quasiconvexity are M-quasiconvexity of orders 
1 and 2, respectively. For any two positive integers $t_{1}<t_{2}$ with $t_{2}$ a multiple of $t_{1}$, it is easy to see that M-quasiconvexity of order $t_{1}$ implies M-quasiconvexity of order $t_{2}$ for a symmetric tensor $\mathcal{F} \in \mathbb{S}^{n^{t_{2} m}}$. To conclude this paper, we state the following result without proof, which generalizes Theorem 4.2.

TheOREM 8.3. If $S$ is a linear subspace of $\mathbb{R}^{n}$, then co-nonnegativity of order $t$ over $S$ implies $M$-quasiconvexity of order $t$ over $S$.

\section{REFERENCES}

[1] A. A. Ahmadi, A. Olshevsky, P. A. Parrilo, And J. N. Tsitsiklis, NP-hardness of deciding convexity of quartic polynomials and related problems, Math. Program., 137 (2013), pp. 453-476.

[2] B. W. Bader and T. G. Kolda, Matlab Tensor Toolbox, Version 2.5, http://www.sandia. gov/ tgkolda/TensorToolbox, 2012.

[3] S. BANACH, Über homogene Polynome in $\left(L^{2}\right)$, Studia Math., 7 (1938), pp. 36-44.

[4] A. Barvinok, A Course in Convexity, Grad. Stud. Math. 54, AMS, Providence, RI, 2002.

[5] G. Blekherman, P. Parrilo, and R. Thomas, Semidefinite Optimization and Convex Algebraic Geometry, MOS-SIAM Ser. Optim. 13, SIAM, Philadelphia, PA, 2012.

[6] R. E. Burkard and E. Cela, Heuristics for biquadratic assignment problems and their computational comparison, European J. Oper. Res., 83 (1995), pp. 283-300.

[7] R. E. Burkard, E. Cela, and B. Klinz, On the biquadratic assignment problem, in P. M. Pardalos and H. Wolkowicz, eds., Quadratic Assignment and Related Problems, DIMACS Ser. Discrete Math. Theoret. Comput. Sci. 16, AMS, Providence, RI, (1994), pp. 117-146.

[8] B. Chen, S. He, Z. LI, AND S. Zhang, Maximum block improvement and polynomial optimization, SIAM J. Optim., 22 (2012), pp. 87-107.

[9] Y. Ding and H. Wolkowicz, A low-dimensional semidefinite relaxation for the quadratic assignment problem, Math. Oper. Res., 34 (2009), pp. 1008-1022.

[10] M. Grant and S. Boyd, CVX: Matlab Software for Disciplined Convex Programming, Version 1.2, http://cvxr.com/cvx, 2010.

[11] S. He, Z. LI, AND S. Zhang, Approximation algorithms for homogeneous polynomial optimization with quadratic constraints, Math. Program., 125 (2010), pp. 353-383.

[12] J. W. Helton and J. Nie, Semidefinite representation of convex sets, Math. Program., 122 (2010), pp. 21-64.

[13] D. Henrion, J. B. Lasserre, and J. Loefberg, GloptiPoly 3: Moments, optimization and semidefinite programming, Optim. Methods Softw., 24 (2009), pp. 761-779.

[14] D. HilberT, Über die Darstellung definiter Formen als Summe von Formenquadraten, Math. Ann., 32 (1888), pp. 342-350.

[15] B. Jiang, S. He, Z. Li, And S. Zhang, Moments tensors, Hilbert's identity, and k-wise uncorrelated random variables. Math. Oper. Res., 39 (2014), pp. 775-788.

[16] B. JiAng, Z. Li, AND S. Zhang, On cones of nonnegative quartic forms, Found. Comput. Math., 17 (2017), pp. 161-197.

[17] E. JondeAu AND M. Rockinger, Optimal portfolio allocation under higher moments, European Finan. Manage., 12 (2006), pp. 29-55.

[18] P. -M. Kleniati, P. Parpas, And B. Rustem, Partitioning Procedure for Polynomial Optimization: Application to Portfolio Decisions with Higher Order Moments, COMISEF Working Papers Series, WPS-023, 2009.

[19] E. DE KLERK AND R. SotiRov, Exploiting group symmetry in semidefinite programming relaxations of the quadratic assignment problem, Math. Program., 122 (2010), pp. 225-246.

[20] T. G. Kolda And B. W. BADER, Tensor decompositions and applications, SIAM Rev., 51 (2009), pp. 455-500.

[21] J. B. LASSERRE, Global optimization with polynomials and the problem of moments, SIAM J. Optim., 11 (2001), pp. 796-817.

[22] M. Laurent, Sums of Squares, Moment Matrices and Optimization over Polynomials, in M. Putinar and S. Sullivant eds., Emerging Applications of Algebraic Geometry, Series, IMA Vol. Math. Appl. 149, Springer, Berlin, 2009.

[23] Z. Li, S. He, and S. Zhang, Approximation Methods for Polynomial Optimization: Models, Algorithms, and Applications, Springer Briefs Optim., Springer, New York, 2012.

[24] L. Lim, Singular values and eigenvalues of tensors: A variational approach, in Proceedings of the 1st IEEE International Workshop on Computational Advances of Multi-tensor Adaptive Processing, IEEE, Piscataway, NJ, 2005, pp. 129-132. 
[25] Z. Luo, J. F. Sturm, And S. Zhang, Multivariate nonnegative quadratic mappings, SIAM J. Optim., 14 (2004), pp. 1140-1162.

[26] Z. Luo And P. Tseng, On the convergence of the coordinate descent method for convex differentiable minimization, J. Optim. Theory Appl., 72 (1992), pp. 7-35.

[27] D. Maringer And P. PARPas, Global optimization of higher order moments in portfolio selection, J. Global Optim., 43 (2009), pp. 219-230.

[28] H. M. Markowitz, Portfolio selection, J. Finance, 7 (1952), pp. 79-91.

[29] T. Mavridou, P. M. Pardalos, L. S. Pitsoulis, and M. G. C. Resende, A GRasP for the biquadratic assignment problem, European J. Oper. Res., 105 (1998), pp. 613-621.

[30] M. Mhiri and J.-L. Prigent, International portfolio optimization with higher moments, Int. J. Econ. Finance, 2 (2010), pp. 157-169.

[31] P. A. PARrilo, Structured Semidefinite Programs and Semialgebraic Geometry Methods in Robustness and Optimization, Ph.D. Dissertation, California Institute of Technology, Pasadena, CA, 2000.

[32] A. Pappas, Y. Sarantopoulos, and A. Tonge, Norm attaining polynomials, Bull. Lond. Math. Soc., 39 (2007), pp. 255-264.

[33] J. Peng, H. Mittelmann, And X. Li, A new relaxation framework for quadratic assignment problems based on matrix splitting, Math. Program. Comput., 2 (2010), pp. 59-77.

[34] M. Putinar and F.-H. Vasilescu, Positive polynomials on semialgebraic sets, C. R. Math. Acad. Sci. Paris, 328 (1999), pp. 585-589.

[35] L. QI, Eigenvalues of a real supersymmetric tensor, J. Symbolic Comput., 40 (2005), pp. 1302-1324.

[36] L. QI, Eigenvalues and invariants of tensors, J. Math. Anal. Appl., 325 (2007), pp. 1363-1377.

[37] B. Reznick, Sums of Even Powers of Real Linear Forms, Mem. Amer. Math. Soc. 96, American Mathematical Society, Providence, RI, 1992.

[38] B. ReZnick, Uniform denominators in Hilbert's seventeenth problem, Math. Z., 220 (1995), pp. $75-97$.

[39] J. F. Sturm And S. Zhang, On cones of nonnegative quadratic functions, Math. Oper. Res., 28 (2003), pp. 246-267.

[40] X. Zhang, C. LING, AND L. QI, The best rank-1 approximation of a symmetric tensor and related spherical optimization problems, SIAM J. Matrix Anal. Appl., 33 (2012), pp. 806-821. 